\title{
Tree-Structured Complementary Filter Banks Using All-Pass Sections
}

\author{
PHILLIP A. REGALIA, STUDENT MEMBER, IEEE, SANJIT K. MITRA, FELLOW, IEEE, \\ P. P. VAIDYANATHAN, MEMBER, IEEE, MARKKU K. RENFORS, MEMBER, IEEE, \\ AND YRJÖ NEUVO, SENIOR MEMBER, IEEE
}

\begin{abstract}
Tree-structured complementary filter banks are developed with transfer functions that are simultaneously all-pass complementary and power complementary. Using a formulation based on unitary transforms and all-pass functions, we obtain analysis and synthesis filter banks which are related through a transposition operation, such that the cascade of analysis and synthesis filter banks achieves an all-pass function. The simplest structure is obtained using a Hadamard transform, which is shown to correspond to a binary tree structure. Tree structures can be generated for a variety of other unitary transforms as well. In addition, given a tree-structured filter bank where the number of bands is a power of two, simple methods are developed to generate complementary filter banks with an arbitrary number of channels, which retain the transpose relationship between analysis and synthesis banks, and allow for any combination of bandwidths. The structural properties of the filter banks are illustrated with design examples, and multirate applications are outlined.
\end{abstract}

\section{INTRODUCTION}

$\mathrm{D}$ ESIGN procedures for computationally efficient uniform filter banks with decimated outputs have received considerable attention in recent years [1]-[12]. Commonly preferred strategies to achieve efficiency include using polyphase techniques or tree-structured architectures [1]. The polyphase technique is useful in multirate applications, as it allows the computational load of the filter bank to be distributed among the branches, whereas the tree-structured architecture affords the possibility of using similar or identical processing techniques within each stage of the tree. In addition, the frequency selectivity of tree-structured filter banks can be understood as a cascade of stages, which affords straightforward design procedures.

The technique of Quadrature Mirror Filtering (QMF), as introduced in [2], allows cancellation of aliasing distortion introduced by critical sampling in multirate systems [1]. A variety of elegant design techniques have been advanced to obtain filter sets which satisfy QMF conditions [3]-[11]. In addition to removing aliasing effects introduced into critically sampled subbands, some of these

Manuscript received July 23, 1986; revised March 16, 1987. This work was supported by the National Science Foundation under Grant MIP 85-08017.

P. A. Regalia and S. K. Mitra are with the Department of Electrical and Computer Engineering, University of California, Santa Barbara, CA 93106.

P. P. Vaidyanathan is with the Department of Electrical Engineering, California Institute of Technology, Pasadena, CA 91125.

M. K. Renfors and Y. Neuvo are with the Department of Electrical Engineering, Tampere University of Technology, SF 33101, Tampere, Finland.

IEEE Log Number 8717302. designs allow for perfect magnitude reconstruction of the original signal [4], [7], [28], perfect phase reconstruction [6], [8], [9], or both [3], [10], [11]. The two-band QMF system has been well studied, and thus it is often convenient to develop mutliple-band extensions from tree-structured architectures of two-band QMF banks. Such filters are quite popular in subband coding of speech [3], [4].

The design of filter banks in TDM-FDM systems has received considerable attention as well [13]-[17]. For example, Claasen and Mecklenbrauker [15] have shown that an efficient transmultiplexer realization can be achieved using a tree structure of two-band filter banks. In addition, the use of branch filtering [18] (or directional filtering) has proved valuable in achieving computational efficiency in transmultiplexer design [16], [17]. Such filtering techniques may be understood as the successive cascade of powercomplementary filter structures, resulting in a power-complementary filter bank.

However, important applications arise for filter banks where the individual filters are desired to have different bandwidths, and where decimation and interpolation of the signals is not desired. For example, in audiosignal processors [19] octave-band filters may be preferred over a uniform filter bank. Similarly, crossover networks for multiple loudspeaker audiosystems typically do not coincide with integer-band filters. Nonetheless, the design of nonuniform filter banks has received sparse attention. Implementation techniques may include cascading low-pass and high-pass filters, or implementing each filter separately, which may not be computationally efficient strategies.

This paper considers the properties of a class of complementary tree-structured filter banks, which can be formulated as unitary transforms operating on all-pass functions. The transfer functions of such filter banks are simultaneously all-pass complementary and power complementary [20], and the structures may be configured to provide an arbitrary number of channels with any desired combination of bandwidths. A framework is presented whereby a synthesis filter bank is obtained systematically from an analysis filter bank using a transposition operation, such that the cascade of analysis and synthesis filter banks achieves an all-pass function. The formulation allows the desired properties to be structurally induced. The application of these complementary filter banks to multirate systems is outlined as well. 


\section{Notations}

Transfer functions for the analysis filter banks are denoted by $\left\{H_{i}(z)\right\}$, while those for the synthesis filter banks are denoted by $\left\{G_{i}(z)\right\}$. All-pass functions are denoted by $\left\{A_{i}(z)\right\}$ or $\left\{a_{i}(z)\right\}$. Vectors and matrices appear in bold type, with superscript $T$ denoting vector or matrix transpose. The tilde notation $(\sim)$ denotes paraconjugate transposition, that is, replacing $z$ with $z^{-1}$, and replacing each coefficient of a transfer function with its complex conjugate. For a transfer vector evaluated along the unit circle, or for a constant matrix, the tilde notation reduces to the conjugate transpose. We denote by $I$ the identity matrix, and by $\boldsymbol{J}$ the permutation matrix with ones along the anti-diagonal (from lower left corner to upper right corner). The dimension of a square matrix is indicated by a subscript (e.g., $I_{2}$ for the $2 \times 2$ identity matrix) if it is not clear from the context. For convenience below, row and column indices for vectors and matrices are numbered starting from 0 rather than 1 .

We consider a bank of $N$ filters $\left\{H_{i}(z)\right\}$ or $\left\{G_{i}(z)\right\}$ such that the following two conditions hold:

(i) The transfer functions are all-pass complementary:

$$
\left|H_{0}\left(e^{j \omega}\right)+H_{1}\left(e^{j \omega}\right)+\cdots+H_{N-1}\left(e^{j \omega}\right)\right|=1 .
$$

(ii) The transfer functions are power complementary:

$$
\left|H_{0}\left(e^{j \omega}\right)\right|^{2}+\left|H_{1}\left(e^{j \omega}\right)\right|^{2}+\cdots+\left|H_{N-1}\left(e^{j \omega}\right)\right|^{2}=1
$$

and likewise for the transfer function set $\left\{G_{i}(z)\right\}$. Transfer function sets which are simultaneously all-pass complementary and power complementary are termed doubly complementary [25]. Equation (1) is important in applications where the output signals from the analysis bank are to be summed directly with no additional synthesis bank filtering, such as in multiple loudspeaker audiosystems. Equation (2) guarantees that where one filter has a passband, the others have a stophand, thereby providing good channel separation. ${ }^{1}$ Note that if the transfer functions $\left\{H_{i}(z)\right\}_{i=0}^{N-1}$ are arranged as the elements of a column vector $\boldsymbol{h}(z)$, then by analytic continuation (2) is equivalent to $\tilde{\boldsymbol{h}}(z) \boldsymbol{h}(z)=1$.

Section III reviews the development of two-band filter banks. Subsequent sections then generalize the results to multiple-band filter banks. Although the development is based in the discrete time domain, the results are equally valid in the continuous time domain upon replacing digital all-pass functions with analog all-pass functions [40].

\section{Two-Band Filter Banks}

For $N=2$, a solution which satisfies the constraints of (1) and (2) is

$$
\left|H_{0}\left(e^{j \omega}\right)+H_{1}\left(e^{j \omega}\right)\right|^{2}=\left|H_{0}\left(e^{j \omega}\right)\right|^{2}+\left|H_{1}\left(e^{j \omega}\right)\right|^{2}=1 .
$$

The left equality in (3) imposes that $H_{0}\left(e^{j \omega}\right)$ and $H_{1}\left(e^{j \omega}\right)$ be in phase quadrature at all frequencies. (This follows by

\footnotetext{
${ }^{1}$ The passband for the $i$ th filter is taken as the frequency range for which $\left|H_{i}\left(e^{. \omega}\right)\right| \approx 1$.
}

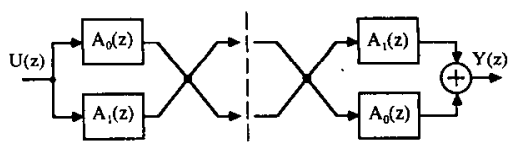

(a)

(b)

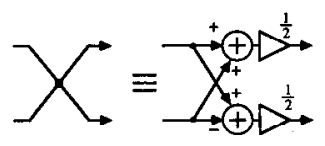

(c)

Fig. 1. Two-band filter banks. (a) Analysis bank. (b) Synthesis bank.

taking $H_{0}\left(e^{j \omega}\right)$ and $H_{1}\left(e^{j \omega}\right)$ as phasors in the complex plane and applying the law of cosines.) As a consequence, both $\left[H_{0}\left(e^{j \omega}\right)+H_{1}\left(e^{j \omega}\right)\right]$ and $\left[H_{0}\left(e^{j \omega}\right)-H_{1}\left(e^{j \omega}\right)\right]$ must generate the same magnitude response, namely unity in view of (3). Thus by analytic continuation we obtain

$$
\begin{aligned}
& H_{0}(z)+H_{1}(z)=A_{0}(z) \\
& H_{0}(z)-H_{1}(z)=A_{1}(z)
\end{aligned}
$$

where $A_{0}(z)$ and $A_{1}(z)$ are all-pass functions. Solving for $H_{0}(z)$ and $H_{1}(z)$ results in

$$
\left[\begin{array}{l}
H_{0}(z) \\
H_{1}(z)
\end{array}\right]=\frac{1}{2}\left[\begin{array}{rr}
1 & 1 \\
1 & -1
\end{array}\right]\left[\begin{array}{l}
A_{0}(z) \\
A_{1}(z)
\end{array}\right]
$$

or, in matrix form,

$$
\boldsymbol{h}(z)=\frac{1}{2} \boldsymbol{R} a(z)
$$

where

and

$$
\boldsymbol{h}(z)=\left[\begin{array}{ll}
H_{0}(z) & H_{1}(z)
\end{array}\right]^{T}
$$

$$
a(z)=\left[\begin{array}{ll}
A_{0}(z) & A_{1}(z)
\end{array}\right]^{T} .
$$

A structural interpretation appears as Fig. 1(a) (where the "criss-cross" denotes a scaled butterfly operation).

Note that the matrix $R / \sqrt{2}$ is orthogonal

$$
\boldsymbol{R}^{T} \boldsymbol{R}=2 \boldsymbol{I}
$$

and may be considered a $2 \times 2$ Hadamard or DFT matrix [30], [41].

Fig. 1(a) represents an analysis bank. The constraints of (3) are easily formed into a synthesis filter bank, too. Letting $G_{0}(z)$ and $G_{1}(z)$ denote the transfer functions of the synthesis filter bank, we define

$$
\left[G_{0}(z) G_{1}(z)\right]=\frac{1}{2}\left[A_{1}(z) A_{0}(z)\right]\left[\begin{array}{rr}
1 & 1 \\
1 & -1
\end{array}\right]
$$

in the form

$$
\boldsymbol{g}^{T}(z)=\frac{1}{2} \boldsymbol{a}^{T}(z) \boldsymbol{J} \boldsymbol{R}^{T}
$$

where

and

$$
\boldsymbol{g}^{T}(z)=\left[\begin{array}{ll}
G_{0}(z) & G_{1}(z)
\end{array}\right]
$$

$$
a^{T}(z) J=\left[\begin{array}{ll}
A_{1}(z) & A_{0}(z)
\end{array}\right]^{T}
$$

with structural interpretation as sketched in Fig. 1(b). The cascade of analysis and synthesis banks achieves an all-pass function:

$$
\begin{aligned}
\boldsymbol{g}^{T}(z) \boldsymbol{h}(z) & =\frac{1}{4} \boldsymbol{a}^{T}(z) \boldsymbol{J} \boldsymbol{R}^{T} \boldsymbol{R a}(z) \\
& =\frac{1}{2} \boldsymbol{a}^{T}(z) \boldsymbol{J a}(z)=A_{0}(z) A_{1}(z) .
\end{aligned}
$$

From (5) and (7) it follows that $G_{0}(z)=H_{0}(z)$, and $G_{1}(z)$ $=-H_{1}(z)$. Thus an equivalent representation of the anal- 


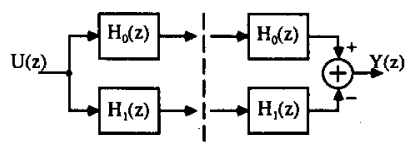

Fig. 2. Equivalent representation of two-band filter bank.

ysis/synthesis system of Fig. 1 is that shown in Fig. 2, where the overall transfer function is

$$
\begin{aligned}
\frac{Y(z)}{U(z)} & =\left[H_{0}(z)\right]^{2}-\left[H_{1}(z)\right]^{2} \\
& =\left[H_{0}(z)+H_{1}(z)\right]\left[H_{0}(z)-H_{1}(z)\right] \\
& =A_{0}(z) A_{1}(z)
\end{aligned}
$$

in agreement with (8).

\section{Additional Properties}

From (5) it follows that

$$
\begin{aligned}
\arg [ & \left.H_{0}\left(e^{j \omega}\right)\right]-\arg \left[H_{1}\left(e^{j \omega}\right)\right] \\
= & \frac{\arg \left[A_{0}\left(e^{j \omega}\right)\right]+\arg \left[A_{1}\left(e^{j \omega}\right)\right]}{2} \\
& -\frac{\arg \left[A_{0}\left(e^{j \omega}\right)\right]-\arg \left[A_{1}\left(e^{j \omega}\right)\right]}{2} \\
= & \pm \pi / 2, \quad \text { for all } \omega .
\end{aligned}
$$

This means that the two transfer functions $H_{0}(z)$ and $H_{1}(z)$ exhibit phase quadrature along the unit circle for any pair of all-pass filters $A_{0}(z)$ and $A_{1}(z)$. The same is also true for the synthesizer transfer functions $G_{0}(z)$ and $G_{1}(z)$. We enumerate a few cases:

Case (i): $A_{0}(z)$ and $A_{1}(z)$ are real all-pass functions (i.e., real-valued for real $z$ ). The representation of (5) is then characteristic of wave lattice filters [21], [22], although direct $z$-domain design techniques have received current attention [23]-[25]. By using filter structures which remain all-pass in spite of coefficient quantization [23], [26], [27], the complementary properties of (3) become structurally induced, and a low passband sensitivity realization of $H_{0}(z)$ and $H_{1}(z)$ is obtained [21]-[25]. In addition, by choosing $H_{0}(z)$ and $H_{1}(z)$ as a symmetric half-band filter pair, the structures of Fig. 1 are well suited for multirate filtering (i.e., QMF) applications [7], [28]. We examine such applications in Section VII.

Case (ii): $A_{0}(z)$ and $A_{1}(z)$ are complex all-pass functions, with $A_{1}(z)=A_{0}^{*}\left(z^{*}\right)$. In this case, (5) can still yield two real doubly complementary transfer functions in a low-sensitivity realization. Design techniques and multirate applications can be found in [28].

Case (iii): $A_{0}(z)$ and $A_{1}(z)$ are arbitrary all-pass functions. In this case, (5) gives rise to two complex doubly complementary transfer functions.

We shall primarily be concerned with case (i) above for simplicity, although most of the subsequent results apply with minor modification to cases (ii) and (iii) as well.

\section{Multiple-Band Filter Banks}

The results obtained above are easily generalized to $N$ transfer functions $\left\{H_{i}(z)\right\}$ or $\left\{G_{i}(z)\right\}$ by choosing $a(z)$ as an $N \times 1$ all-pass function vector, and $\boldsymbol{R}$ as an $N \times N$ unitary matrix. In particular, we shall consider Hadamard and DFT matrices for $\boldsymbol{R}$, although the subsequent results are valid for virtually any choice of unitary matrix.

For the Hadamard matrix, higher order matrices $(N \times$ $N)$ are obtained through [30]-[32]:

$$
\boldsymbol{R}_{N}=\boldsymbol{R}_{N / 2} \otimes \boldsymbol{R}_{2}
$$

where $\otimes$ denotes the Kronecker product of matrices, and $\boldsymbol{R}_{2}$ is the $2 \times 2$ Hadamard matrix as given in (5). In practice $N$ is limited to a power of 2 . Similarly, DFT matrices of dimension $N \times N$ are defined by

$$
\begin{aligned}
\boldsymbol{R}_{N} & =\left[\begin{array}{ccccc}
1 & 1 & 1 & \cdots & 1 \\
1 & W_{N} & W_{N}^{2} & \cdots & W_{N}^{N-1} \\
\vdots & \vdots & \vdots & \ddots & \vdots \\
1 & W_{N}^{N-1} & W_{N}^{2(N-1)} & \cdots & W_{N}^{(N-1)(N-1)}
\end{array}\right] \\
W_{N} & =e^{-j(2 \pi / N)}
\end{aligned}
$$

It is often convenient to choose $N$ as a power of 2 , allowing fast radix-2 FFT algorithms to implement the transform of (12). Both the matrices of (11) and (12) satisfy

$$
\tilde{\boldsymbol{R}} \boldsymbol{R}=N \boldsymbol{I}_{N}
$$

where $I_{N}$ is the $N \times N$ identity matrix.

By choosing the all-pass function vector $a(z)=\left[A_{0}(z)\right.$ $\left.A_{1}(z) \cdots A_{N-1}(z)\right]^{T}$, we can define $N$ transfer functions $\boldsymbol{h}(z)=\left[\begin{array}{lll}H_{0}(z) & H_{1}(z) \cdots H_{N-1}(z)\end{array}\right]^{T}$ according to

$$
\boldsymbol{h}(z)=\frac{1}{N} \boldsymbol{R}_{N} \boldsymbol{a}(z) \text {. }
$$

Similarly, $N$ synthesis filter bank transfer functions $\boldsymbol{g}^{T}(z)$ $=\left[G_{0}(z) \quad G_{1}(z) \cdots G_{N-1}(z)\right]$ can be defined by

$$
\boldsymbol{g}^{T}(z)=\frac{1}{N} \boldsymbol{a}^{T}(z) J \tilde{\boldsymbol{R}}_{N} .
$$

The doubly-complementary relations of the $N$ transfer functions $\left\{H_{i}(z)\right\}$ in (14) are readily shown. From (14) we obtain

$$
\tilde{\boldsymbol{R}} \boldsymbol{h}(z)=\boldsymbol{a}(z) .
$$

If we denote the column vectors of $\boldsymbol{R}$ by $\left\{\boldsymbol{c}_{i}\right\}$, then (16) corresponds to $N$ scalar equations of the form

$$
\tilde{\boldsymbol{c}}_{i} \boldsymbol{h}(z)=A_{i}(z), \quad i=0,1, \ldots, N-1 .
$$

Equation (17) identifies $N$ distinct all-pass complementary summations of the transfer functions $\left\{H_{i}(z)\right\}$. In particular, if

$$
c_{i}=\left[\begin{array}{lll}
1 & 1 & \cdots
\end{array}\right]^{T}, \quad \text { for some } i
$$

then (1) is satisfied. Hadamard, DFT', and complex BIFORE [34] matrices, for example, all satisfy (18). The power complementary property along the unit circle follows from (13):

$$
\begin{aligned}
\sum_{i=0}^{N-1}\left|H_{i}\left(e^{j \omega}\right)\right|^{2} & =\tilde{\boldsymbol{h}}\left(e^{j \omega}\right) \boldsymbol{h}\left(e^{j \omega}\right)=\frac{1}{N^{2}} \tilde{\boldsymbol{a}}\left(e^{j \omega}\right) \tilde{\boldsymbol{R}} \boldsymbol{R} \boldsymbol{a}\left(e^{j \omega}\right) \\
& =\frac{1}{N} \tilde{\boldsymbol{a}}\left(e^{j \omega}\right) \boldsymbol{a}\left(e^{j \omega}\right) \\
& =\frac{1}{N} \sum_{i=0}^{N-1}\left|A_{i}\left(e^{j \omega}\right)\right|^{2}=1
\end{aligned}
$$




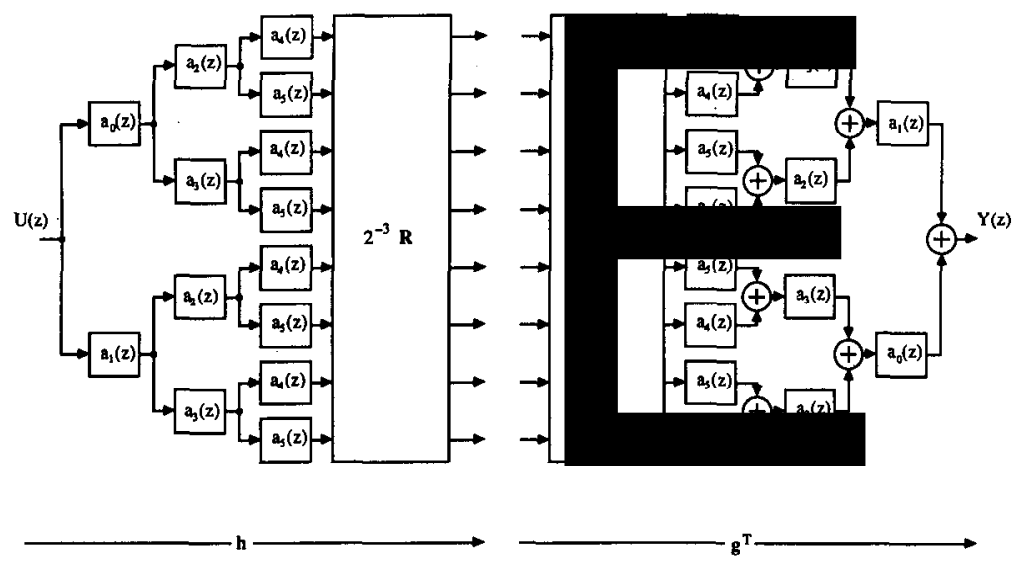

Fig. 3. Illustrating a binary tree structure for the all-pass vector.

Similar results can be verified for the transfer functions $\left\{G_{i}(z)\right\}$ of the synthesis filter bank.

Note from (14) and (15) that the cascade of analysis and synthesis filter banks yields an overall transfer function

$$
\begin{aligned}
\boldsymbol{g}^{T}(z) \boldsymbol{h}(z) & =\frac{1}{N} \boldsymbol{a}^{T}(z) \boldsymbol{J a}(z) \\
& =\frac{1}{N} \sum_{i=0}^{N-1} A_{i}(z) A_{N-1-i}(z)
\end{aligned}
$$

which is not all pass unless each term in the summation of (20) is identical. Some remedies are as follows:

(i): In (15) the all-pass function vector $\boldsymbol{a}^{T}(z) J$ can be replaced with $b^{T}(z)=\left[B_{0}(z) \quad B_{1}(z) \cdots B_{N-1}(z)\right]$, such that

$$
B_{i}(z)=\frac{\operatorname{lcm}\left(A_{0}(z), A_{1}(z), \ldots, A_{N-1}(z)\right)}{A_{i}(z)}
$$

where $\operatorname{lcm}(\cdot)$ denotes the least common multiple of the arguments. This method guarantees an overall transfer function $\boldsymbol{g}^{T}(z) \boldsymbol{h}(z)=\operatorname{lcm}\left(A_{0}(z), A_{1}(z), \ldots, A_{N-1}(z)\right)$. However, this brute force remedy may lead to $b(z)$ of high order if the elements of $a(z)$ are relatively prime.

(ii): To circumvent this problem, we can restrict the all-pass function vector $a(z)$ to derive from a binary tree structure of all-pass filters, as illustrated in Fig. 3 with a 3 -stage tree. For an $n$-stage tree, $\boldsymbol{a}(z)$ is recognized as the (left) Kronecker product of $n 2 \times 1$ all-pass function vectors:

$$
a(z)=\left[\begin{array}{l}
a_{2 n-2}(z) \\
a_{2 n-1}(z)
\end{array}\right] \otimes\left[\begin{array}{l}
a_{2 n-4}(z) \\
a_{2 n-3}(z)
\end{array}\right] \otimes \cdots \otimes\left[\begin{array}{l}
a_{0}(z) \\
a_{1}(z)
\end{array}\right]
$$

and thus

$$
\begin{aligned}
& \boldsymbol{a}^{T}(z) \boldsymbol{J}=\left[a_{2 n-1}(z) \quad a_{2 n-2}(z)\right] \\
& \otimes\left[a_{2 n-3}(z) \quad a_{2 n-4}(z)\right] \\
& \otimes \cdots \otimes\left[a_{1}(z) \quad a_{0}(z)\right] .
\end{aligned}
$$

With this choice of all-pass function vector, the cascade of analysis and synthesis banks becomes an all-pass function:

$$
\begin{aligned}
\boldsymbol{g}^{T}(z) \boldsymbol{h}(z)= & \frac{1}{N} \boldsymbol{a}^{T}(z) \boldsymbol{J a}(z) \\
= & \frac{1}{N}\left(\left[a_{2 n-1}(z) a_{2 n-2}(z)\right]\right. \\
& \left.\otimes \cdots \otimes\left[a_{1}(z) a_{0}(z)\right]\right) \\
& \cdot\left(\left[\begin{array}{c}
a_{2 n-2}(z) \\
a_{2 n-1}(z)
\end{array}\right] \otimes \cdots \otimes\left[\begin{array}{l}
a_{0}(z) \\
a_{1}(z)
\end{array}\right]\right) \\
= & \prod_{i=0}^{2 n-1} a_{i}(z)
\end{aligned}
$$

where we have used the algebra of Kronecker products [29]. In this fashion, a synthesis bank is systematically obtained from an analysis bank using a transposition operation. The motivation behind (21) is that the Kronecker product composition of the all-pass vector arises naturally from a binary tree structure of two-band filter banks, as illustrated next.

\section{Structural Interpretations}

The above discussion is applicable when the number of bands is a power of two, i.e., $N=2^{n}$. We shall exemplify structures for eight-band $\left(N=2^{3}\right)$ Hadamard and DFT analysis filter banks, since the extension to other powersof-two is straightforward. The corresponding synthesis filter banks are obtained using a (conjugatc) transposition operation, followed by reversing the all-pass vector.

From the definition of Hadamard matrices in (11), and the Kronecker product composition of the all-pass vector in (21), we obtain for an eight-band analysis filter bank:

$$
\begin{aligned}
\boldsymbol{h}(z)= & \frac{1}{8} \boldsymbol{R} \boldsymbol{a}(z) \\
= & \frac{1}{8}\left(\left[\begin{array}{rr}
1 & 1 \\
1 & -1
\end{array}\right] \otimes\left[\begin{array}{rr}
1 & 1 \\
1 & -1
\end{array}\right] \otimes\left[\begin{array}{rr}
1 & 1 \\
1 & -1
\end{array}\right]\right) \\
& \cdot\left(\left[\begin{array}{l}
a_{4}(z) \\
a_{5}(z)
\end{array}\right] \otimes\left[\begin{array}{l}
a_{2}(z) \\
a_{3}(z)
\end{array}\right] \otimes\left[\begin{array}{l}
a_{0}(z) \\
a_{1}(z)
\end{array}\right]\right) \\
= & \frac{1}{8}\left(\left[\begin{array}{rr}
1 & 1 \\
1 & -1
\end{array}\right]\left[\begin{array}{l}
a_{4}(z) \\
a_{5}(z)
\end{array}\right]\right) \otimes\left(\left[\begin{array}{rr}
1 & 1 \\
1 & -1
\end{array}\right]\left[\begin{array}{l}
a_{2}(z) \\
a_{3}(z)
\end{array}\right]\right) \\
& \otimes\left(\left[\begin{array}{rr}
1 & 1 \\
1 & -1
\end{array}\right]\left[\begin{array}{l}
a_{0}(z) \\
a_{1}(z)
\end{array}\right]\right) .
\end{aligned}
$$


The last step follows directly from the algebra of Kronecker products [29]. The structural interpretation of (23) is shown in Fig. 4, in the form of a tree structure of two-band filter banks. This is a natural consequence of the Kronecker product composition of both the Hadamard matrix and the all-pass function vector.

In a similar manner, by permuting the rows of an $8 \times 8$ DFT matrix in bit-reversed order, a sparse matrix factorization can be obtained as:
(21), tree-structured filter banks can be developed for a variety of unitary transforms, all of which yield powercomplementary transfer functions due to the inherent satisfaction of (13) for unitary transforms.

\section{Filter Design}

Assuming the all-pass filters are real, it is clear that using the Hadamard transform (or any real unitary trans-

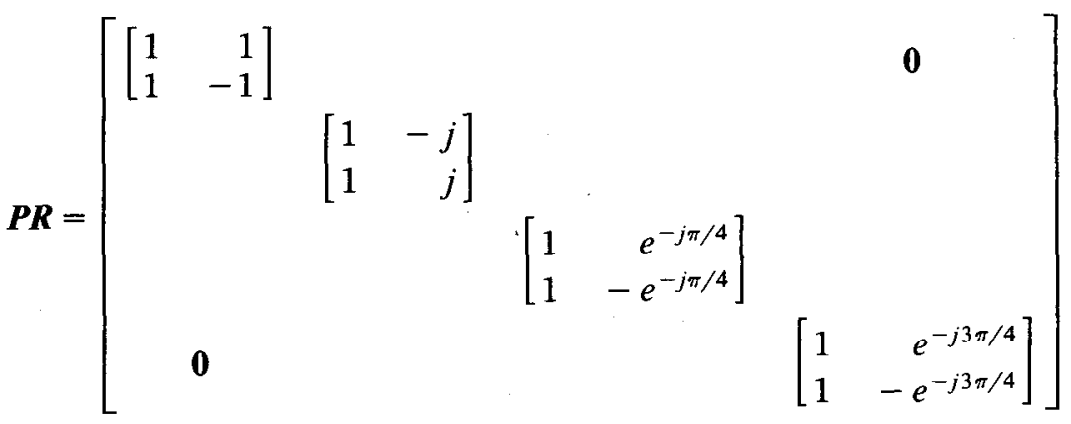

$$
\begin{aligned}
& \cdot\left[\begin{array}{cc}
I_{2} \otimes\left[\begin{array}{rr}
1 & 1 \\
1 & -1
\end{array}\right] & \mathbf{0} \\
0 & I_{2} \otimes\left[\begin{array}{rr}
1 & -j \\
1 & j
\end{array}\right]
\end{array}\right]\left[\mathbf{I}_{4} \otimes\left[\begin{array}{rr}
1 & 1 \\
1 & -1
\end{array}\right]\right]
\end{aligned}
$$

where $\boldsymbol{P}$ is the bit-reversed ordering permutation matrix. Equation (24) corresponds to a decimation-in-frequency radix-2 algorithm. By combining this with (21), an equivalent representation can be obtained as

$$
\begin{aligned}
& \boldsymbol{P h}(z)=\frac{1}{8} \boldsymbol{P R} \boldsymbol{a}(z)
\end{aligned}
$$

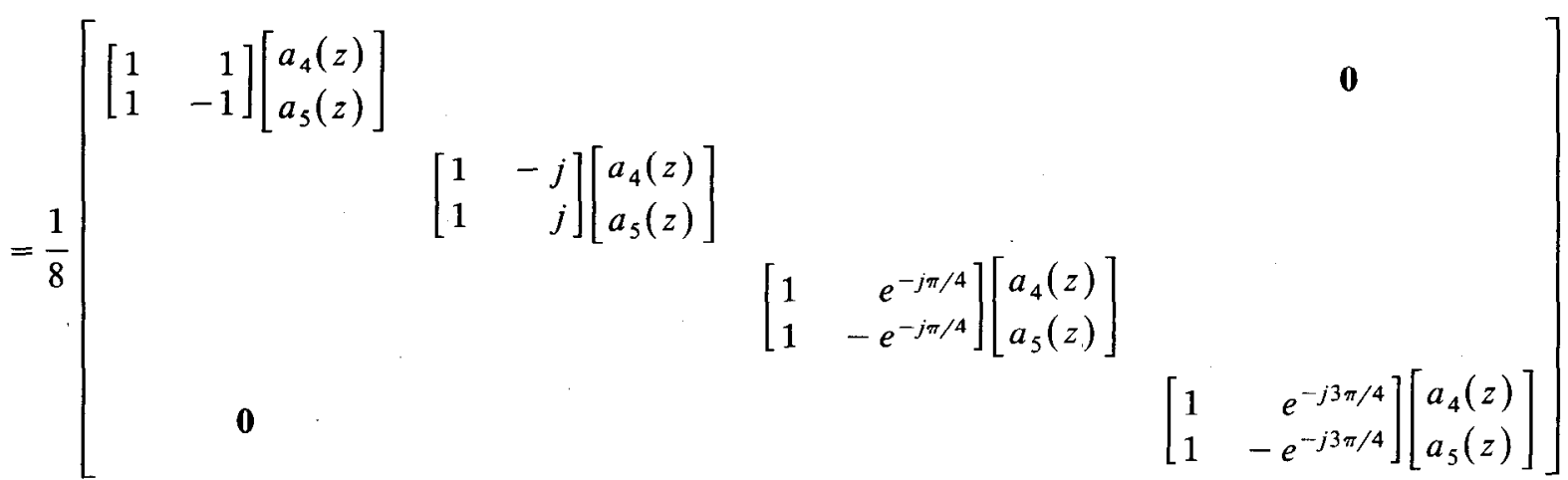

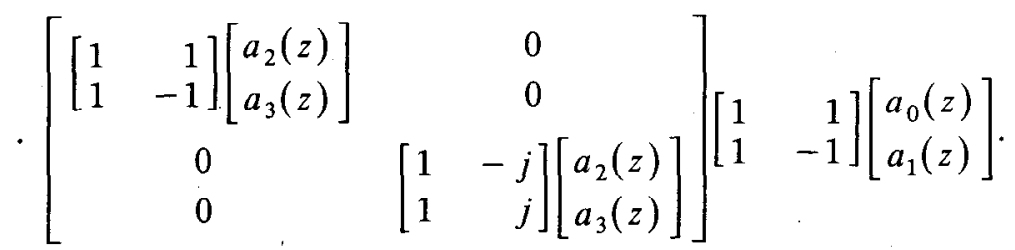

The overall structure is that of Fig. 5, which may be interpreted as a modified "pruned" FFT flowgraph. Note that if all complex multiplications are set to unity, Fig. 5 reduces to the Hadamard filter bank of Fig. 4 (upon renumbering the transfer functions).

We point out here that, for a variety of unitary transforms, sparse matrix factorizations analogous to (24) are available [30]-[35], in direct correspondence with fast transform algorithms. Thus with the Kronecker product (i.e., tree-structured) composition of the all-pass vector in form) in (14) and (15) yields $N$ real transfer functions for the analysis and synthesis banks whereas using the DFT gives $N$ complex transfer functions. In most applications this is a drawback of complex transforms, even though an $N / 2$-channel real bank can be obtained from the complex bank by combining the complex-conjugate channels. It is also seen that complex arithmetic is needed for implementing the latter stages of the tree-structured DFT filter bank of Fig. 5. Therefore, we shall concentrate in the following on filter banks based on the Hadamard transform. 


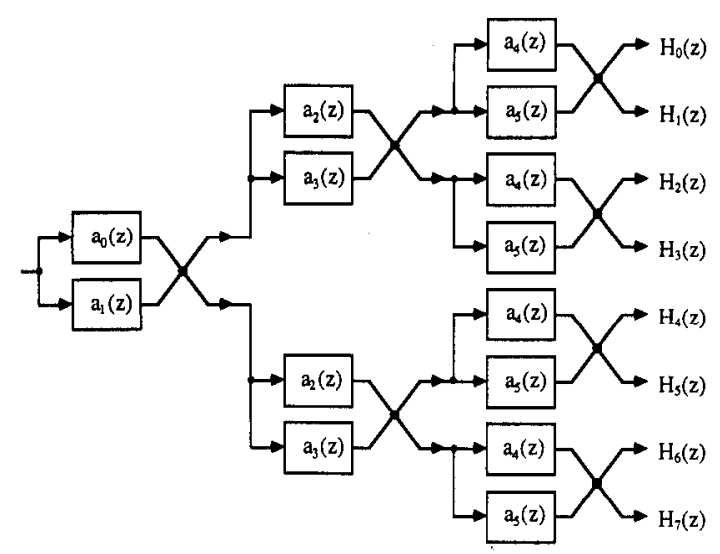

Fig. 4. Eight-band Hadamard analysis bank.

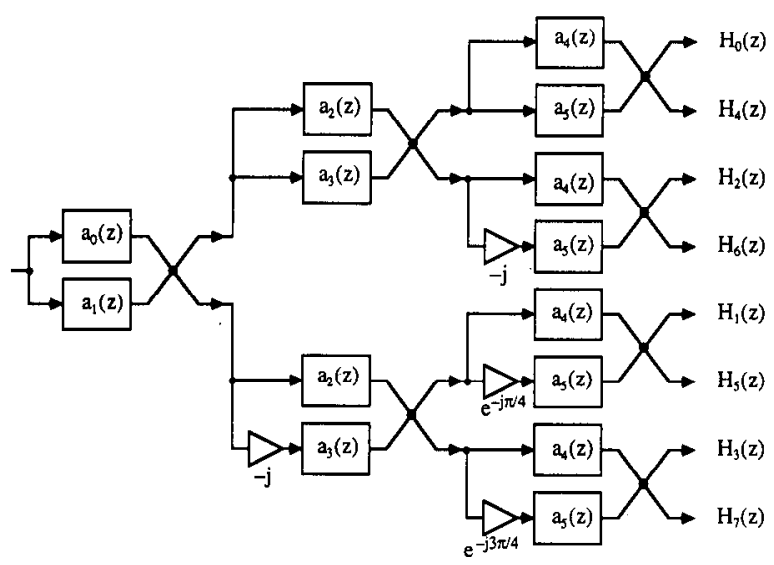

Fig. 5. Eight-band DFT bank.

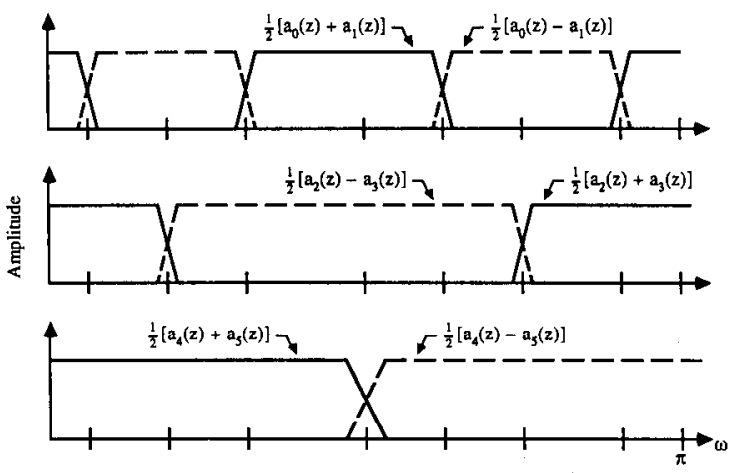

Fig. 6. Characteristic amplitude responses for the complementary filter pairs of an eight channel Hadamard bank.

Let us consider tree-structured Hadamard filter banks of the type of Fig. 4. The channel frequency responses of the overall filter bank can be expressed in terms of the frequency responses of the complementary filter pairs of the form $\frac{1}{2}\left[a_{2 i-2}(z) \pm a_{2 i-1}(z)\right]$ appearing at each stage. Each filter pair shapes some of the transition bands of the overall bank. If the $N=2^{n}$ channels of the overall bank are designed to have distinct passbands, the $N-1$ distinct transition bands must be shaped by the $n$ filter pairs. It is then clear that, for $N>2$, some of these filter pairs must be multiband designs. One possible set of amplitude responses for the filter pairs are sketched in Fig. 6 for an eight-channel bank. If the same approach is used for higher $N$, one of the filter pairs must have $N / 2$ transition bands. Notice that the ordering of the filter stages is completely free. Therefore, it is advantageous to order the filter pairs in the analysis bank according to increasing overall order. This means that the filter pair with highest overall order is realized by $a_{0}(z)$ and $a_{1}(z)$ (cf. Fig. 4).

It is well known (see, e.g., [22], [25]) that classical (e.g., elliptic) odd-order low-pass filters are realizable as the parallel connection of two all-pass filters. Such designs can be used for the low-pass/high-pass filter pairs in the tree structure. A bandpass/bandstop filter pair can be obtained through a low-pass-to-bandpass transformation of a low-pass prototype. However, the multiband specifications needed in the eight-channel bank of Fig. 6 are more difficult to satisfy. For example, the multiband magnitude response corresponding to $\frac{1}{2}\left[a_{0}(z) \pm a_{1}(z)\right]$ can be obtained by applying a low-pass-to-bandpass transformation to a bandpass/bandstop filter pair. In this case though, there is control of the locations of all the transition bands but the transition bandwidths cannot be controlled independently.

For filter banks with $N>8$, the design of filter pairs providing the required multiband amplitude responses becomes very difficult. For example, the repeated application of low-pass-to-bandpass transformations does not provide independent control on the locations of all the transition bands. In the following we develop alternative filter bank structures with an arbitrary number of channels. Using these techniques, it is possible to realize doubly complementary filter banks using only low-pass designs for the filter pairs of each stage.

\section{Filter Banks with an Arbitrary Number OF BANDS}

The previous section has developed tree-structured filter banks for which the number of bands is a power of two, i.e., $N=2^{n}$, for some integer $n$. The temptation now is to extend the formulation to an arbitrary number of bands. One approach to obtaining an arbitrary number of bands is to combine an appropriate number of outputs (inputs) of a $2^{n}$-band doubly-complementary analysis (synthesis) filter bank. For example, a three-band filter bank can be realized by combining two bands of a four-band filter bank. More generally, the process of generating a filter bank with $M\left(<2^{n}\right)$ new transfer functions $\left\{H_{i}^{\prime}(z)\right\}$ or $\left\{G_{i}^{\prime}(z)\right\}$ by appropriately combining $N=2^{n}$ transfer functions $\left\{H_{i}(z)\right\}$ or $\left\{G_{i}(z)\right\}$ suggests the matrix equation

$$
\boldsymbol{h}^{\prime}(z)=\operatorname{Th}(z)=\frac{1}{N} \operatorname{TRa}(z)
$$

for the analysis bank, and

$$
\boldsymbol{g}^{\prime T}(z)=g^{T}(z) \tilde{\boldsymbol{T}}=\frac{1}{N} \boldsymbol{a}^{T}(z) \boldsymbol{J} \tilde{\boldsymbol{R}} \tilde{\boldsymbol{T}}
$$

for the synthesis bank, for some $M \times N$ matrix $T$, as sketched in Fig. 7. In (26) we have assumed that the synthesis bank and analysis bank retain a transpose rela- 


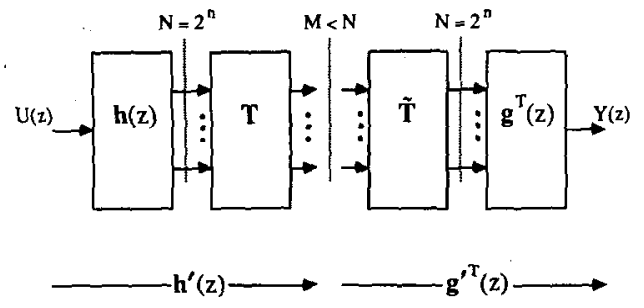

Fig. 7. Generating a filter bank with fewer than $N$ bands.

tionship, which will be justified in the discussion that follows.

The transfer functions $\left\{H_{i}(z)\right\}$ can always be grouped such that the all-pass complementary relation of (1) is obeyed among the new transfer functions $\left\{H_{i}^{\prime}(z)\right\}$. Hence, we investigate under what conditions the power complementary relation of (2) is satisfied among the $\left\{H_{i}^{\prime}(z)\right\}$. Using (26a), the sum of magnitude squares of the $\left\{H_{i}^{\prime}(z)\right\}$ becomes

$$
\begin{aligned}
\sum_{i=0}^{M-1}\left|H_{i}^{\prime}\left(e^{j \omega}\right)\right|^{2} & =\tilde{\boldsymbol{h}}^{\prime}\left(e^{j \omega}\right) \boldsymbol{h}^{\prime}\left(e^{j \omega}\right) \\
& =\frac{1}{N^{2}} \tilde{\boldsymbol{a}}\left(e^{j \omega}\right) \tilde{\boldsymbol{R}} \tilde{\boldsymbol{T}} \boldsymbol{T} \boldsymbol{R} \boldsymbol{a}\left(e^{j \omega}\right) .
\end{aligned}
$$

Let

$$
\tilde{\boldsymbol{T}} \boldsymbol{T}=I+Q
$$

where $I$ is the $N \times N$ identity matrix. Then (27) becomes

$$
\begin{aligned}
\sum_{i=0}^{M-1}\left|H_{i}^{\prime}\left(e^{j \omega}\right)\right|^{2}= & \frac{1}{N^{2}} \tilde{\boldsymbol{a}}\left(e^{j \omega}\right) \tilde{\boldsymbol{R}} \boldsymbol{I R} \boldsymbol{a}\left(e^{j \omega}\right) \\
& +\frac{1}{N^{2}} \tilde{\boldsymbol{a}}\left(\boldsymbol{e}^{j \omega}\right) \tilde{\boldsymbol{R}} \boldsymbol{Q R} \boldsymbol{R}\left(e^{j \omega}\right),
\end{aligned}
$$

i.e.,

$$
\sum_{i=0}^{M-1}\left|H_{i}^{\prime}\left(e^{j \omega}\right)\right|^{2}=1+\frac{1}{N^{2}} \tilde{\boldsymbol{h}}\left(e^{j \omega}\right) \boldsymbol{Q h}\left(e^{j \omega}\right)
$$

This becomes unity if and only if $\tilde{h}(z) Q h(z)=0$. For specific values of the $Q$ matrix, this can be accomplished by imposing appropriate conditions on the components $H_{i}(z)$ of the vector $\boldsymbol{h}(z)$. For example, let $M=N-1$, and let $T$ in $(26)$ be $(N-1) \times N$ of the form

$$
\boldsymbol{T}=\left[\begin{array}{ccccc}
1 & 0 & \cdots & 0 & 0 \\
0 & 1 & & 0 & 0 \\
\vdots & & \ddots & & \vdots \\
0 & 0 & \cdots & 1 & 1
\end{array}\right]
$$

which corresponds to

$$
H_{i}^{\prime}(z)= \begin{cases}H_{i}(z), & i=0,1, \ldots, N-3 \\ H_{N-2}(z)+H_{N-1}(z), & i=N-2\end{cases}
$$

The $N \times N$ matrix $Q$ is now

$$
Q=\left[\begin{array}{cccc}
0 & \cdots & & 0 \\
0 & \ddots & & \vdots \\
\vdots & & 0 & 1 \\
0 & \cdots & 1 & 0
\end{array}\right]
$$

where $(29 b)$ yields

$$
\begin{aligned}
\sum_{i=0}^{M-1}\left|H_{i}^{\prime}\left(e^{j \omega}\right)\right|^{2}= & +\frac{1}{N^{2}}\left[\tilde{H}_{N-2}(z) H_{N-1}(z)\right. \\
& \left.+\tilde{H}_{N-1}(z) H_{N-2}(z)\right]\left.\right|_{z=e^{j \omega}}
\end{aligned}
$$

If $H_{N-1}(z)$ and $H_{N-2}(z)$ are restricted to be such that

$$
\tilde{H}_{N-2}(z) H_{N-1}(z)+\tilde{H}_{N-1}(z) H_{N-2}(z)=0
$$

then the set of $M$ transfer functions $\left\{H_{i}^{\prime}(z)\right\}$ indced satisfies the power-complementary property. Equation (34) states that the numerator of $\tilde{H}_{N-1}(z) H_{N-2}(z)$ must be a conjugate antisymmetric polynomial, or equivalently, that $H_{N-1}\left(e^{j \omega}\right)$ and $H_{N-2}\left(e^{j \omega}\right)$ be in phase quadrature for all $\omega$.

It can be easily seen from these discussions that, given $N$ transfer functions $\left\{H_{i}(z)\right\}$ satisfying the doubly complementary property, it is possible to obtain $M(<N)$ transfer functions $\left\{H_{i}^{\prime}(z)\right\}$ which again satisfy the doubly complementary property, simply by constraining appropriate pairs in $h(z)$ to be in phase quadrature and adding such pairs.

This procedure naturally brings about the following question: How severe is the phase-quadrature restriction, from the viewpoint of realizing practically useful transfer functions? It will be recalled from Section III that the two-band filter banks of (5) and (7) yield transfer functions which exhibit phase quadrature along the unit circle, independent of the choice of the all-pass filters. Thus given a tree-structure of such two-band filter banks, one can systematically identify transfer function pairs in phase quadrature. To complete the question, we note that two IIR transfer functions (with the same denominator) exhibit phase quadrature along the unit circle if their numerators are linear phase with the same center of (anti)symmetry and of opposite parity (i.e., one a symmetric polynomial, the other antisymmetric). This by itself imposes only mild restrictions on the realizable magnitude responses (e.g., an antisymmetric polynomial must have a zero at $z=1$ [4], [24]).

An alternate viewpoint can establish matrix properties of $\boldsymbol{T}$ such that the power complementary property of $\boldsymbol{h}^{\prime}(z)$ or $\boldsymbol{g}^{\prime}(z)$, as well as the all-pass property of $\boldsymbol{g}^{\prime T}(z) \boldsymbol{h}^{\prime}(z)$, remain intact (cf. Fig. 7). We state these results in the following lemmas.

Lemma 1: With $h^{\prime}(z)=T h(z)$ as per (26a), let $\tilde{T} T=I$ $+\boldsymbol{Q}$. Define $\boldsymbol{S} \triangleq \tilde{R} Q R$ in (29a). If $S$ is jointly symmetric and centro-skew-symmetric (i.e., symmetric along the main diagonal and skew-symmetric along the antidiagonal), then the elements of $h^{\prime}(z)$ are power complementary, i.e., $\tilde{\boldsymbol{h}}^{\prime}(z) \boldsymbol{h}^{\prime}(z)=1$.

The proof is in the Appendix. We remark here that the joint symmetric and centro-skew-symmetric property implies that

$$
\boldsymbol{J S} \boldsymbol{J}=-\boldsymbol{S}
$$

or, since $\boldsymbol{J}^{2}=\boldsymbol{I}$, then equivalently $\boldsymbol{J} \boldsymbol{S}=-\boldsymbol{S} \boldsymbol{J}$. The properties of such matrices are quite analogous to those of doubly symmetric matrices [36], [37]. 


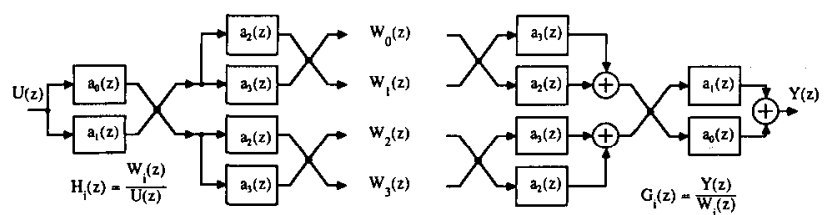

Fig. 8. Four-band Hadamard filter bank for Example 1.

Lemma 2: With $T$ satisfying the constraints of Lemma $1, \boldsymbol{g}^{\prime T}(z) \boldsymbol{h}^{\prime}(z)$ is a scalar all-pass function if $\boldsymbol{g}^{T}(z) \boldsymbol{h}(z)$ is. Proof: From (26) we have

$$
\boldsymbol{g}^{\prime T}(z) \boldsymbol{h}^{\prime}(z)=\frac{1}{N} \boldsymbol{a}^{T}(z) \boldsymbol{J} \tilde{\boldsymbol{R}} \tilde{\boldsymbol{T}} \boldsymbol{T R a}(z) .
$$

Substituting $\tilde{\boldsymbol{T} T} \boldsymbol{T}=\boldsymbol{I}+\boldsymbol{Q}$ and applying the result of (22), we have

$$
\mathbf{g}^{\prime T}(z) \boldsymbol{h}^{\prime}(z)=\prod_{i=0}^{2 n-1} a_{i}(z)+\frac{1}{N} \boldsymbol{a}^{T}(z) J \boldsymbol{S a}(z)
$$

We now show $\boldsymbol{a}^{T}(z) \boldsymbol{J S a}(z)=0$. To see this, note that

$$
[\boldsymbol{J S}]^{T}=\boldsymbol{S} \boldsymbol{J}=-\boldsymbol{J} \boldsymbol{S}
$$

that is, $\boldsymbol{J S}$ is skew-symmetric. As such, by invoking a standard result for skew-symmetric matrices, $\boldsymbol{a}^{T}(z)$ - $\boldsymbol{J S a}(z)=0$, and the lemma is proved.

From the above two lemmas, it is clear that, given a doubly complementary analysis/synthesis filter bank pair, one can modify both analysis and synthesis banks to obtain new doubly complementary filter banks, such that the transfer function of the cascade remains invariant.

\section{Illustrative EXamples}

This section illustrates the above concepts from a structural viewpoint. We include some simple design examples to enumerate the different possibilities available within the same structural framework.

\section{Example 1}

By extending Fig. 1 into a tree structure, a four-band filter bank shown in Fig. 8 is obtained. Following analysis similar to Section IV, the transfer functions for the analysis bank are found to be

$$
\boldsymbol{h}(z)=\frac{1}{4}\left(\left[\begin{array}{rr}
1 & 1 \\
1 & -1
\end{array}\right]\left[\begin{array}{l}
a_{2}(z) \\
a_{3}(z)
\end{array}\right]\right) \otimes\left(\left[\begin{array}{rr}
1 & 1 \\
1 & -1
\end{array}\right]\left[\begin{array}{l}
a_{0}(z) \\
a_{1}(z)
\end{array}\right]\right)
$$

or, writing out explicitly,

$$
\left[\begin{array}{l}
H_{0}(z) \\
H_{1}(z) \\
H_{2}(z) \\
H_{3}(z)
\end{array}\right]=\frac{1}{4}\left[\begin{array}{rrrr}
1 & 1 & 1 & 1 \\
1 & -1 & 1 & -1 \\
1 & 1 & -1 & -1 \\
1 & -1 & -1 & 1
\end{array}\right]\left[\begin{array}{l}
a_{0}(z) a_{2}(z) \\
a_{0}(z) a_{3}(z) \\
a_{1}(z) a_{2}(z) \\
a_{1}(z) a_{3}(z)
\end{array}\right]
$$

We thus obtain a four-band doubly complementary Hadamard filter bank. Similar results apply to the four transfer functions $\left\{G_{i}(z)\right\}$ of the corresponding synthesis filter bank, where $G_{0}(z)=H_{0}(z), G_{1}(z)=-H_{1}(z), G_{2}(z)$ $=-H_{2}(z)$, and $G_{3}(z)=H_{3}(z)$.

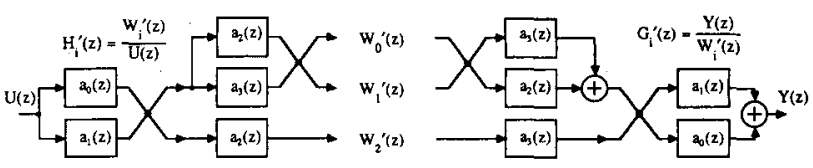

Fig. 9. Resulting three-band filter bank obtained from that of Fig. 7 by "collapsing" one butterfly connection.

Next, we combine two outputs (inputs) of the analysis (synthesis) filter bank to obtain a three-band filter bank. To maintain the power complementary relation in the three-band filter bank, we must combine transfer functions which exhibit phase quadrature along the unit circle. Let $H_{0}^{\prime}(z)=H_{0}(z), \quad H_{1}^{\prime}(z)=H_{1}(z), \quad H_{2}^{\prime}(z)=H_{2}(z)+H_{3}(z)$ for the analysis bank. Then

$$
\left[\begin{array}{l}
H_{0}^{\prime}(z) \\
H_{1}^{\prime}(z) \\
H_{2}^{\prime}(z)
\end{array}\right]=\left[\begin{array}{llll}
1 & 0 & 0 & 0 \\
0 & 1 & 0 & 0 \\
0 & 0 & 1 & 1
\end{array}\right]\left[\begin{array}{l}
H_{0}(z) \\
H_{1}(z) \\
H_{2}(z) \\
H_{3}(z)
\end{array}\right]
$$

in the form

$$
h^{\prime}(z)=\operatorname{Th}(z)
$$

Note that

$$
\sum_{i=0}^{2} H_{i}^{\prime}(z)=\sum_{i=0}^{3} H_{i}(z)=a_{0}(z) a_{2}(z)
$$

that is, the all-pass complementary property is left intact. The power complementary property follows by noting that $H_{2}(z)$ and $H_{3}(z)$ are the outputs of a two-band filter bank in the second stage of the tree, and thus exhibit phase quadrature for $z=e^{j \omega}$. Fig. 9 shows the resulting threeband analysis and synthesis filter banks, which effectively is obtained from that of Fig. 8 by "collapsing" one of the two-point butterflies in the second stage of the tree structure. From Fig. 9 it is easily verified that the overall transfer function from analysis bank input to synthesis bank output is

$$
\frac{Y(z)}{U(z)}=a_{0}(z) a_{1}(z) a_{2}(z) a_{3}(z)
$$

just as for the four-band structure of Fig. 8. Note that these results can also be verified from the lemmas of the previous section, although for this example greater intuition is gained from inspection of the structure.

An alternate interpretation of Fig. 9 begins with a two-band filter as in Fig. 1. One output of the analysis bank is split into two bands, while the other output passes through an all-pass filter. Note that this interpretation is precisely that which leads to "compensation" paths as introduced in other works [3], [5], [7] on non-power-of-two tree-structured filter banks. By applying this idea again to the three-band structure of Fig. 9, we obtain additional four-band doubly complementary filter banks in Fig. 10 . Clearly, the pattern may be continued to obtain an arbitrary number of bands. We point out that, as three pairs of all-pass filters are present in Fig. 10, either structure may be obtained from a $8 \times 8$ Hadamard filter bank through 


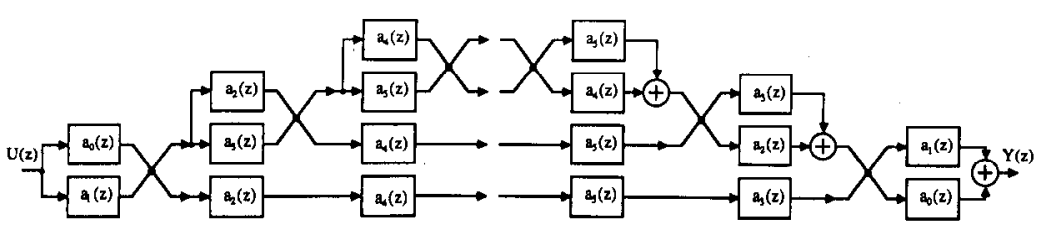

(a)

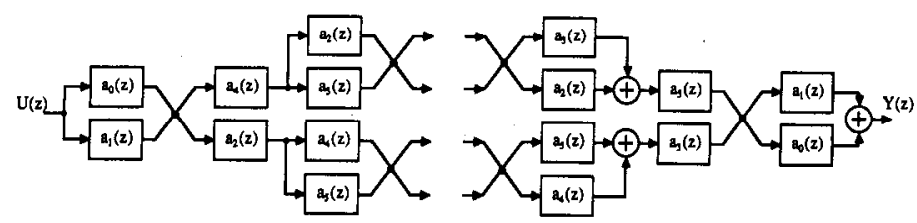

(b)

Fig. 10. Alternate four-band doubly complementary filter banks.

the appropriate combination of transfer functions. The relative merits of the different possibilities are briefly examined next.

\section{Example 2}

In this example we compare different design strategies for a three-band analysis filter bank. In particular, we constrain the transition frequencies between adjacent passbands to lie at $0.2 \pi$ and $0.7 \pi$, with $40-\mathrm{dB}$ minimum stopband attenuation in each band. Upon choosing the structure of Fig. 9, two design strategies present themselves:

(i) Choose $a_{0}(z)$ and $a_{1}(z)$ such that their sum and difference form a bandpass/bandstop complementary filter pair with half-power frequencies for either response (i.e., transition frequencies) at $0.2 \pi$ and $0.7 \pi$. The bandstop output is then split into low-pass and high-pass outputs by choosing $a_{2}(z)$ and $a_{3}(z)$ such that their sum and difference form a low-pass/high-pass complementary pair.

(ii) Choose $a_{0}(z)$ and $a_{1}(z)$ to form a low-pass/highpass complementary filter pair with transition frequency at $0.2 \pi$. The high-pass output is then split into bandpass and high-pass outputs by choosing $a_{2}(z)$ and $a_{3}(z)$ to form a low-pass/high-pass complementary filter pair with a transition frequency of $0.7 \pi$.

For design (i), we choose a sixth-order bandpass filter, derived from a third-order elliptic low-pass prototype. The stopband rejection is chosen at $40 \mathrm{~dB}$, and by choosing a passband ripple of $0.00043 \mathrm{~dB}$, the complementary response also exhibits $40-\mathrm{dB}$ stopband rejection. To split the bandstop output into low-pass and high-pass outputs, we choose $a_{2}(z)$ and $a_{3}(z)$ such that their sum and difference form a fifth-order elliptic low-pass/high-pass complementary pair, with $40-\mathrm{dB}$ stopband attenuation for either response. The choice of fifth order is to ensure adequate separation of the two passbands of the bandstop transfer function from the first stage of the tree. To obtain the best separation of these passbands, the transition band for the low-pass/high-pass pair of the second stage should be properly "centered" between the two passbands of the bandstop filter. If we let $\omega_{1}$ and $\omega_{2}$ denote the half-power frequencies of the bandpass filter of the first stage, the half-power frequency $\omega_{3}$ of the low-pass/high-pass pair of

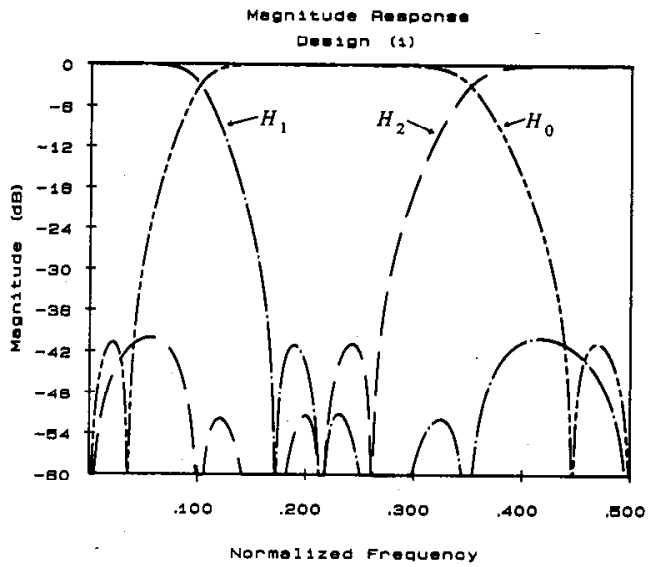

Fig. 11. Individual magnitude responses for Example 2, design (i).

TABLE I

POLE LOCATIONS FOR DESIGN (i)

\begin{tabular}{lcc}
\hline & $\omega_{1}=0.2 \pi, \omega_{2}=0.7 \pi$ & \\
& $\omega_{3}=2 \tan ^{-1} \sqrt{\tan \left(\omega_{1} / 2\right) \tan \left(\omega_{2} / 2\right)}=0.3742 \pi$ & \\
\hline Filter Pair & Digilal Pules & Allpass Functions \\
\hline Bandpass- & $z=0.00$ & $a_{0}(z)$ \\
Bandstop & 0.2214 & \\
pair & $-0.4380 \pm j 0.6090$ & $a_{1}(z)$ \\
$\omega_{1}, \omega_{2}$ & $0.6592 \pm j 0.4742$ & $a_{2}(z)$ \\
Lowpass- & $0.1353 \pm j 0.4524$ & $a_{3}(z)$ \\
Highpass & $0.1872 \pm j 0.8106$ & \\
pair & 0.1120 & \\
$\omega_{3}$ & & \\
\hline
\end{tabular}

the second stage should be chosen according to

$$
\omega_{3}=2 \tan ^{-1} \sqrt{\tan \left(\omega_{1} / 2\right) \tan \left(\omega_{2} / 2\right)} .
$$

The all-pass filters are easily found by matching their poles to those of the desired transfer functions, in accordance with the design procedures discussed in [22], for example. The poles of the elliptic transfer functions described above can be found via the program reported in [38], or through bilinearly transformed analog filter design equations (see [22] for more detail in this regard). The pole locations for the all-pass filters of this example are listed in Table I. Fig. 11 shows the magnitude responses for the 


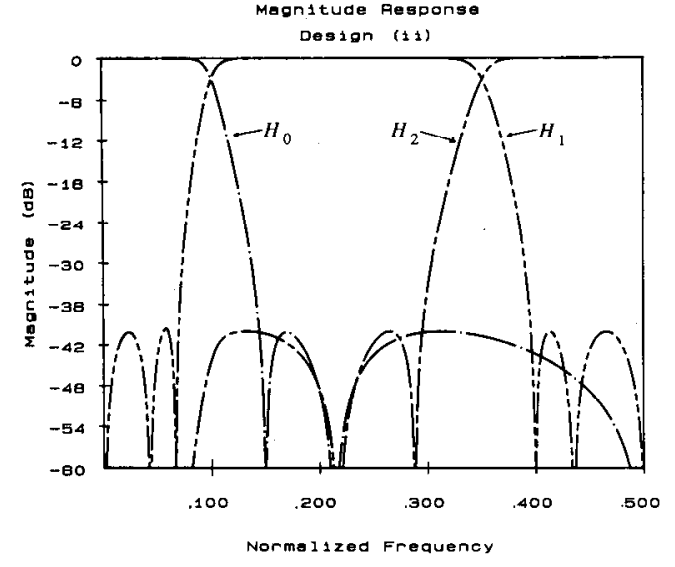

Fig. 12. Individual magnitude responses for design (ii) of Example 2.

TABLE II

Polf Iocations FOR DFsign (ii)

\begin{tabular}{lcc}
\hline Filter Pair & \multicolumn{1}{c}{ Digital Poles } & Allpass Functions \\
\hline Lowpass- & $z=0.5852 \pm j 0.3223$ & $a_{0}(z)$ \\
Highpass & 0.5095 & $a_{1}(z)$ \\
pair \#1 & $0.7298 \pm j 0.5204$ & \\
$\omega_{1}$ & $-0.3849 \pm j 0.4019$ & $a_{2}(z)$ \\
Lowpass- & -0.3249 & $a_{3}(z)$ \\
Highpass & $-0.5107 \pm j 0.6906$ & \\
pair \#2 & & \\
$\omega_{2}$ & \\
\hline
\end{tabular}

three bands. The overall order of the analysis filter bank (i.e., the sum of the all-pass filter orders) is 13.

For design (ii), $a_{0}(z)$ and $a_{1}(z)$ are chosen such that their sum and difference form a fifth-order lowpass/high-pass complementary pair with $40-\mathrm{dB}$ minimum stopband attenuation, and a transition frequency of $0.2 \pi$. $a_{2}(z)$ and $a_{3}(z)$ are similarly chosen, but with a transition frequency of $0.7 \pi$. The pole locations for these all-pass filters are listed in Table II. The individual magnitude responses are plotted in Fig. 12, and are seen to exhibit less overlap due to the sharper transition bands, yet the overall filter order is now only 12 . Note also the more consistent stopband ripples compared to Fig. 11. Observe that the low-frequency response of $\mathrm{H}_{2}\left(e^{j \omega}\right)$ drops off quite rapidly for frequencies below $0.2 \pi$. This effect occurs because for $\omega<0.2 \pi, H_{2}\left(e^{j \omega}\right)$ receives attenuation from both stages of the tree. The overall phase response $\arg \left[H_{0}\left(e^{j \omega}\right)+H_{1}\left(e^{j \omega}\right)+H_{2}\left(e^{j \omega}\right)\right]$ appears smoother for design (i) though, as seen from the group delay plot of Fig. 13.

$$
\left[\begin{array}{ccc}
H_{0}(z) & H_{1}(z) & \cdots \\
H_{0}\left(z W_{N}^{-1}\right) & H_{1}\left(z W_{N}^{-1}\right) & \cdots \\
\vdots & \vdots & \\
H_{0}\left(z W_{N}^{-N+1}\right) & H_{1}\left(z W_{N}^{-N+1}\right) & \cdots
\end{array}\right.
$$

An interesting property of the structures of Figs. 9 and 10 (and their multiband extensions) is that, by following

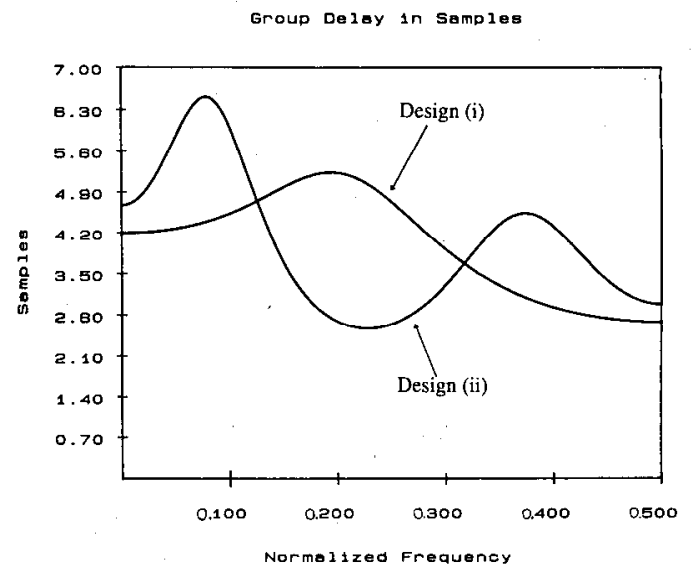

Fig. 13. Comparison of group delay responses between designs (i) and (ii).

design strategies similar to design (ii) above, one can realize multiband filter banks using only low-pass-highpass doubly complementary filter pairs. With such an arrangement, each transition frequency may be varied independently by adjusting the corresponding all-pass filters [39] while maintaining the doubly complementary relation of the filter bank transfer functions.

\section{Multirate Structures}

In this section we explore the usefulness of the filter banks developed in previous sections in systems where the analysis filter bank output signals are to be decimated, as such systems are quite popular, for example, in subband coding [1], [3], [4]. We consider the $N$-band filter bank system of Fig. 14. The output signals from the analysis filter bank are maximally decimated by a factor of $N$, coded and transmitted to the synthesis filter bank, where the signals are decoded, upsampled by a factor of $N$, and then filtered into a composite output signal $\hat{X}(z)$.

In the absence of coding errors, the most general expression for the output signal $\hat{X}(z)$ is

$$
\hat{X}(z)=\frac{1}{N} \sum_{l=0}^{N-1} X\left(z W_{N}^{-l}\right) \sum_{k=0}^{N-1} H_{k}\left(z W_{N}^{-l}\right) G_{k}(z)
$$

where $W_{N}=e^{-j 2 \pi / N}$. Each term $X\left(z W_{N}^{-l}\right), l \neq 0$, represents an aliased image of the input signal. Aliasing distortion is absent from the output signal $\hat{X}(z)$ if and only if the following set of relations holds:

$$
\left.\begin{array}{c}
H_{N-1}(z) \\
H_{N-1}\left(z W_{N}^{-1}\right) \\
\vdots \\
H_{N-1}\left(z W_{N}^{-N+1}\right)
\end{array}\right]\left[\begin{array}{c}
G_{0}(z) \\
G_{1}(z) \\
\vdots \\
G_{N-1}(z)
\end{array}\right]=\left[\begin{array}{c}
T(z) \\
0 \\
\vdots \\
0
\end{array}\right]
$$

in the form

$$
\boldsymbol{H}_{N}(z) \boldsymbol{g}(z)=T(z) \boldsymbol{e}_{1}
$$




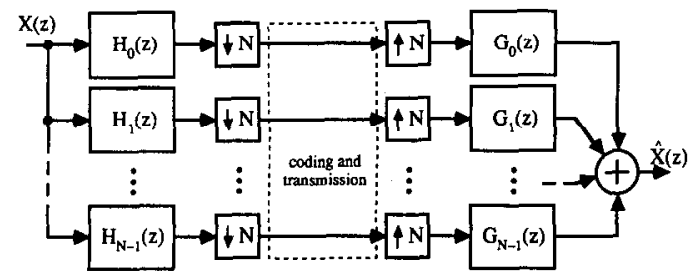

Fig. 14. $N$-band maximally decimated multirate system.

where $e_{1}$ is the unit vector with a "l" in the first position, and $T(z) / N$ is the overall transfer function of the (aliasfree) multirate system. The matrix $\boldsymbol{H}_{N}(z)$ is termed the aliasing component (AC) matrix corresponding to $h(z)$ [12]. The subscript on the $A C$ matrix will denote both the matrix dimension and the decimation factor of the analysis filter bank output signals.

Given a set of analysis filter bank transfer functions $\left\{H_{i}(z)\right\}$, one might attempt to invert the AC matrix in (44) to solve for a set of synthesis filter bank transfer functions $\left\{G_{i}(z)\right\}$ to obtain an alias-free multirate system. However, general procedures for inverting the $\mathrm{AC}$ matrix (if indeed its inverse exists) are not well established. To simplify the problem, let us make the nontrivial assumption that $\boldsymbol{H}_{N}(z)$ is paraunitary:

$$
\tilde{\boldsymbol{H}}_{N}(z) \boldsymbol{H}_{N}(z)=\boldsymbol{H}_{N}(z) \tilde{\boldsymbol{H}}_{N}(z)=\boldsymbol{I}_{N}, \quad \text { for all } z .
$$

In this case, inversion of the AC matrix in (44) is particularly simple:

$$
\mathbf{g}(z)=T(z) \tilde{\boldsymbol{H}}_{N}(z) \boldsymbol{e}_{1} .
$$

However, by writing explicitly the paraconjugate transpose as

$$
\tilde{\boldsymbol{H}}_{N}(z)=\boldsymbol{H}^{T *}\left(1 / z^{*}\right)
$$

then it is clear that with $\boldsymbol{h}(z)$ a stable recursive analysis bank transfer vector, $g(z)$ obtained via (46) is not guaranteed to be stable.

Greater insight into stability is gained from the relation

$$
\tilde{\boldsymbol{H}}_{N}(z)=\boldsymbol{H}_{N}^{-1}(z)=\frac{\operatorname{adj} \boldsymbol{H}_{N}(z)}{\operatorname{det} \boldsymbol{H}_{N}(z)} .
$$

The term $\operatorname{adj} \boldsymbol{H}_{N}(z)$ causes no stability problems; it is division by det $\boldsymbol{H}_{N}(z)$ which places poles outside the unit circle in the $z$-plane. Now, with $\boldsymbol{H}_{N}(z)$ a stable paraunitary matrix, one can show [10] that $\operatorname{det} \boldsymbol{H}_{N}(z)$ is a stable all-pass function. Consider then the choice

$$
T(z)=N \operatorname{det} \boldsymbol{H}_{N}(z) .
$$

This results in

$$
\boldsymbol{g}(z)=N \operatorname{adj} \boldsymbol{H}_{N}(z) \boldsymbol{e}_{1}
$$

which is a stable synthesis filter bank transfer vector if $\boldsymbol{h}(z)$ is stable. In particular, from (46) we have

$$
G_{i}(z)=T(z) H_{i}^{*}\left(1 / z^{*}\right), \quad \text { for all } i
$$

which, with stability established, implies

$$
\left|G_{i}\left(e^{j \omega}\right)\right|^{2}=N^{2}\left|H_{i}\left(e^{j \omega}\right)\right|^{2}, \quad \text { for all } i, \omega
$$

because $T(z) / N$ is an all-pass function. In summary, we have the following result.

Theorem 1: Given a set of $N$ analysis filter bank transfer functions $\left\{H_{i}(z)\right\}$, if the AC matrix $H_{N}(z)$ in (44) is paraunitary, then a set of stable synthesis filter bank transfer functions $\left\{G_{i}(z)\right\}$ satisfying (52) exists such that (i) aliasing distortion is absent from the output of the maximally decimated multirate system of Fig 14, and (ii) the overall transfer function is all pass.

Corollary 1: Let aliasing distortion and amplitude errors be absent from the maximally decimated multirate system of Fig. 14. If the analysis and synthesis filter bank transfer functions are related as per (51), where $T(z) / N$ is a stable all-pass function, the $\mathrm{AC}$ matrix $\boldsymbol{H}_{N}(z)$ is paraunitary.

Proof: The vector counterpart of (51) is $\mathrm{g}^{T}(z)=$ $T(z) \tilde{h}(z)$. In view of the AC matrix formulation of (44), absence of aliasing distortion is equivalent to the following set of conditions:

$$
\begin{aligned}
\boldsymbol{g}^{T}(z) \boldsymbol{h}\left(z W_{N}^{k}\right)=T(z) \tilde{h}(z) \boldsymbol{h}\left(z W_{N}^{k}\right) & =0, \\
k & =1,2, \ldots, N-1 .
\end{aligned}
$$

The overall transfer function is then

$$
\frac{1}{N} g^{T}(z) h(z)=\frac{1}{N} T(z) \tilde{h}(z) h(z)=\text { all pass. }
$$

Since $T(z) / N$ is all pass and $\tilde{\boldsymbol{h}}(z) \boldsymbol{h}(z)$ is real-valued along the unit circle, then (54) implies $\tilde{h}(z) h(z)=1$ for all $z$. Now, as (53) and (54) are required to hold for all $z$, they continue to hold if $z$ is replaced by $z W_{N}, z W_{N}^{2}$, etc. As such, the constraints of (53) and (54) can be collected to form

$$
\tilde{\boldsymbol{h}}\left(z W_{N}^{l}\right) \boldsymbol{h}\left(z W_{N}^{k}\right)=\left\{\begin{array}{ll}
1, & k=l, \\
0, & \text { otherwise, }
\end{array} \text { for all } z .\right.
$$

Equation (55) may be interpreted as an orthonormality relation between the row vectors of $\boldsymbol{H}_{N}(z)$. Hence satisfaction of (55) is equivalent to paraunitariness of $\boldsymbol{H}_{N}(z)$.

Q.E.D.

The significance of Theorem 1 is explored in greater detail in [10] in the context of FIR filter banks having perfect reconstruction properties. For the present we explore IIR filter banks. Accordingly, we assume that aliasing cancellation and perfect magnitude reconstruction are desired; any constraints on phase reconstruction are relaxed.

Let us revisit the two-band filter banks of Section III, where

$$
\boldsymbol{h}(z)=\left[\begin{array}{l}
H_{0}(z) \\
H_{1}(z)
\end{array}\right]=\frac{1}{2}\left[\begin{array}{rr}
1 & 1 \\
1 & -1
\end{array}\right]\left[\begin{array}{l}
a_{0}(z) \\
a_{1}(z)
\end{array}\right]=\frac{1}{2} \boldsymbol{R}_{2} a(z) .
$$

With the outputs decimated by a factor of 2 , the aliasing component matrix is

$$
\boldsymbol{H}_{2}(z)=\left[\begin{array}{c}
\boldsymbol{h}^{T}(z) \\
\boldsymbol{h}^{T}(-z)
\end{array}\right]=\frac{1}{2}\left[\begin{array}{c}
\boldsymbol{a}^{T}(z) \\
\boldsymbol{a}^{T}(-z)
\end{array}\right] \boldsymbol{R}_{2}^{T}
$$




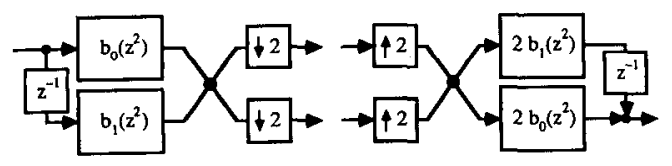

(a)

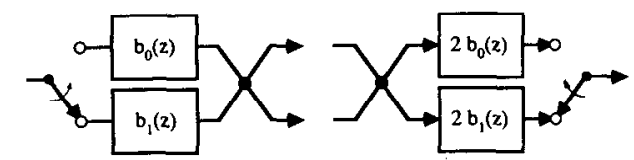

(b)

Fig. 15. (a) Two-band QMF bank. (b) Equivalent polyphase implementation.

whence

$$
H_{2}(z) \tilde{H}_{2}(z)=\frac{1}{2} A_{2}(z) \tilde{A}_{2}(z)
$$

where $\boldsymbol{A}_{2}(z)$ is the $A C$ matrix corresponding to the all-pass vector $\boldsymbol{a}(z)$. Consider the following choice of all-pass functions:

$$
a_{0}(z)=b_{0}\left(z^{2}\right), \quad a_{1}(z)=z^{-1} b_{1}\left(z^{2}\right)
$$

where $b_{0}(\cdot)$ and $b_{1}(\cdot)$ are all-pass functions. In effect, $a_{0}(z)$ and $a_{1}(z)$ are even and odd functions of $z$, respectively, so that $H_{1}(z)=H_{0}(-z)$. This results in

$$
A_{2}(z) \tilde{A_{2}}(z)=2 I_{2}
$$

which, when substituted into (58), reveals that $\boldsymbol{H}_{2}(z)$ is paraunitary. We then have for the synthesis filter bank

$$
g(z)=2 \operatorname{adj} H_{2}(z) e_{1}=2\left[\begin{array}{c}
H_{1}(-z) \\
-H_{0}(-z)
\end{array}\right]=2\left[\begin{array}{c}
H_{0}(z) \\
-H_{1}(z)
\end{array}\right]
$$

and for the overall transfer function

$$
\frac{T(z)}{2}=\operatorname{det} \boldsymbol{H}_{2}(z)=z^{-1} b_{1}\left(z^{2}\right) b_{2}\left(z^{2}\right)=a_{1}(z) a_{2}(z) .
$$

The results of (61) and (62) agree with those obtained in Section III, save for a scale factor of 2 introduced into the synthesis bank transfer functions to offset the loss in signal energy caused by the decimation operation. The choice of all-pass functions in (59) is known to produce symmetric half-band filters in the analysis and synthesis filter banks, and the application of such filters to multirate systems has been observed previously [4], [7], [28]. The structural realization appears as in Fig. 15(a) which, through appropriate exploitation of multirate identities [1], leads to the polyphase structure of Fig. 15(b). Observe that the back-to-back cascade of the two butterfly operations is a transparent connection (save for a scale factor error for $1 / 2$ ). In effect, the aliasing cancellation and perfect magnitude reconstruction properties are tied to the structure of the all-pass vector $\boldsymbol{a}(z)$, and not to $\boldsymbol{R}_{2}$.

Consider now the (four-band) tree structure extension of Fig. 16(a). The cascade of $\boldsymbol{R}$ and $\tilde{\boldsymbol{R}}$ is a transparent connection, and by successive application of the two-band QMF result, it can be verified that the multirate system is free from aliasing effects, and that the overall transfer

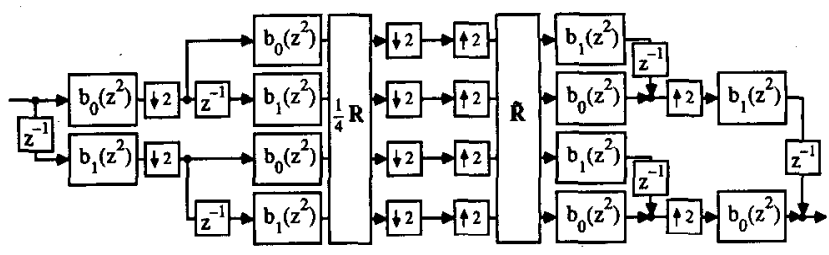

(a)

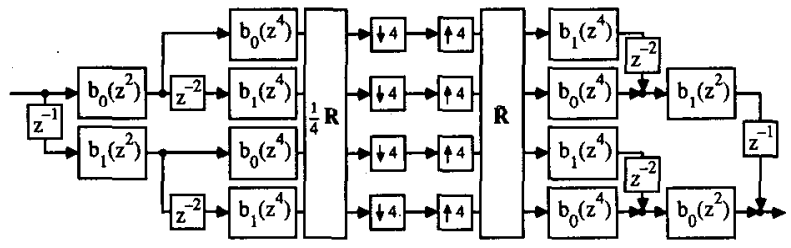

(b)

Fig. 16. (a) Tree-structured QMF bank. (b) Equivalent four-band QMF bank.

function is all pass. By moving all the decimators (resp. interpolators) to the output (input) of the analysis (synthesis) filter bank, the equivalent system of Fig. 16(b) is obtained. By extending the pattern to an $n$-stage tree, an equivalent $2^{n}$-band analysis filter bank obeys the formulation

$$
\begin{aligned}
\boldsymbol{h}(z)= & \frac{1}{N} \boldsymbol{R}_{N} \boldsymbol{a}(z) \\
= & \frac{1}{N} \boldsymbol{R}_{N}\left[\begin{array}{c}
b_{2 n-2}\left(z^{2^{n}}\right) \\
z^{-2^{n-1} b_{2 n-1}}\left(z^{2^{n}}\right)
\end{array}\right] \otimes \cdots \otimes\left[\begin{array}{c}
b_{2}\left(z^{4}\right) \\
z^{-2} b_{3}\left(z^{4}\right)
\end{array}\right] \\
& \cdot\left[\begin{array}{c}
b_{0}\left(z^{2}\right) \\
z^{-1} b_{1}\left(z^{2}\right)
\end{array}\right]
\end{aligned}
$$

where we have allowed the possibility that different stages of the tree may use different all-pass function pairs. Some properties will be discusșed for such tree structures, using the four-band structure of Fig. 16 to illustrate the concepts. In particular, let $\boldsymbol{a}(z)$ and $\boldsymbol{a}^{T}(z) J$ denote the all-pass function vectors for the analysis and synthesis filter banks, respectively. These vectors satisfy

$$
\boldsymbol{a}^{T}(z) \boldsymbol{J}=\frac{1}{N} T(z) \tilde{\boldsymbol{a}}(z)
$$

with

$$
\frac{1}{N} T(z)=z^{2^{n}-1} \prod_{i=0}^{n-1} b_{2 i}\left(z^{2^{i}}\right) b_{2 i+1}\left(z^{2^{i}}\right) .
$$

This follows since, for any all-pass function $a(z)$ :

$$
\tilde{a}(z)=\frac{1}{a(z)}
$$

Hence, (64) states that the $i$ th all-pass function for the synthesis bank is obtained from $T(z) / N$ by dividing out the $i$ th analysis bank all-pass function, which is evident from the tree construction of the all-pass vector in both analysis and synthesis filter banks. Finally, with $\boldsymbol{h}(z)=$ $(1 / N) \boldsymbol{R}_{N} \boldsymbol{a}(z)$ and $\boldsymbol{g}^{T}(z)=\boldsymbol{a}^{T}(z) \boldsymbol{J} \tilde{\boldsymbol{R}}_{N},(64)$ is equivalent to

$$
g^{T}(z)=T(z) \tilde{h}(z)
$$




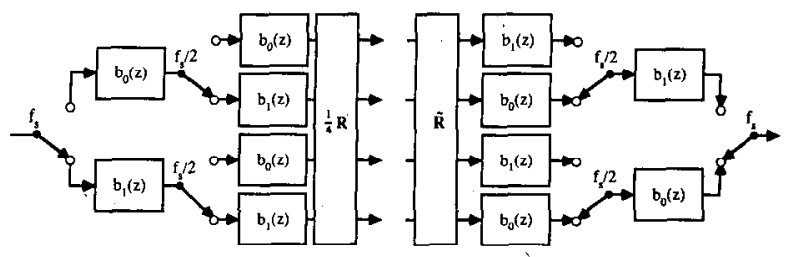

Fig. 17. Hybrid polyphase tree-structured QMF bank.

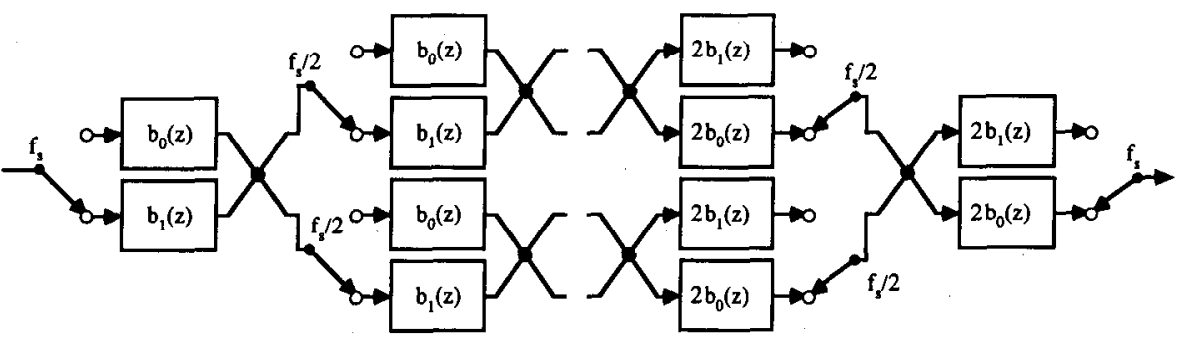

Fig. 18. Equivalent structure to Fig. 17 for a Hadamard QMF bank.

which is the vector counterpart of (51). Since $T(z) / N$ is an all-pass function, the analysis filter bank $h(z)$ of (63) has a paraunitary $A C$ matrix by Corollary 1 .

Having established the aliasing cancellation and perfect magnitude reconstruction results above, let us examine some familiar examples in the present context. We begin with a four-band filter bank:

$$
\boldsymbol{h}(z)=\frac{1}{4} \operatorname{Ra}(z)
$$

where

$$
\boldsymbol{a}(z)=\left[\begin{array}{l}
a_{2}\left(z^{2}\right) \\
a_{3}\left(z^{2}\right)
\end{array}\right] \otimes\left[\begin{array}{l}
a_{0}(z) \\
a_{1}(z)
\end{array}\right] .
$$

Following (59), let us choose for the all-pass functions

$$
a_{0}(z)=a_{2}(z)=b_{0}\left(z^{2}\right), \quad a_{1}(z)=a_{3}(z)=z^{-1} b_{1}\left(z^{2}\right) \text {. }
$$

The resulting four-band filter bank is that of Fig. 16(b). By distributing the decimators and interpolators into the analysis and synthesis banks, the hybrid polyphase tree structure of Fig. 17 is obtained. If the matrix $\boldsymbol{R}$ is a Hadamard matrix, then the equivalent polyphase realization of Fig. 18 is obtained, although similar structures can be obtained for $\boldsymbol{R}$ chosen as some other unitary matrix. In particular, consider choosing $\boldsymbol{R}$ as a column permuted Haar matrix: ${ }^{2}$

$$
\boldsymbol{R}=\left[\begin{array}{cccc}
1 & 1 & 1 & 1 \\
1 & -1 & 1 & -1 \\
\sqrt{2} & 0 & -\sqrt{2} & 0 \\
0 & \sqrt{2} & 0 & -\sqrt{2}
\end{array}\right]
$$

with $a(z)$ as per (67), the structural interpretation of Fig. 19(a) can be obtained. Note that the magnitude filtering functions in the lowest two branches are identical. As such, we expect some simplifications may be possible. Using multirate signal flowgraph manipulation, the path from node $w_{1}$ to node $w_{2}$ can be redrawn as Fig. 19(b), which in

\footnotetext{
${ }^{2}$ Such matrices are also termed Modified Walsh Hadamard Transform (MWHT) matrices [30].
}

turn is equivalent to Fig. 19(c). By inserting Fig. 19(c) into Fig. 19(a), the maximally decimated octave-band filter bank of Fig. 19(d) results. In a similar fashion, by choosing $R$ as an $2^{n} \times 2^{n}$ Haar or MWHT matrix, structural rearrangement of the filter bank system can result in an $(n+1)$-band maximally decimated octave-band filter bank.

\section{CONCLUDING REMARKS}

This paper has considered the properties of a class of doubly complementary filter banks, with the structures subjected to two constraints. First, the analysis and synthesis banks are related through a transposition operation, and second, the cascade of analysis and synthesis filter banks achieves an all-pass function. We have demonstrated how these constraints lead naturally to tree-structured filter banks. The two-band filter banks of Section III form useful building blocks in all structures, and we have shown how various tree-structured extensions may be interpreted as Hadamard, Haar, and DFT filter banks. A1though tree structures of two-band filter banks result in power-of-two structures, simple techniques have been developed to realize doubly complementary filter banks with an arbitrary number of channels. One attractive feature offered is that any desired frequency selectivity may be obtained using only lowpass designs in each stage of the tree. Thus filter banks with independent adjustment of the transition frequencies between adjacent bands become possible, using tunable filter building blocks [39].

The difficulties in filter design for the direct binary tree structure can be overcome by using low-pass/ high-pass (or bandstop/bandstop) filter pairs. We have seen already that in the three-channel case, there are many alternatives for designing the filter pairs appearing in the tree structure. The comparison of these alternatives, as well as the design of doubly complementary filter pairs with a prescribed type of multiband response, is a topic for future research. It should be noted that, in the case of maximally decimated uniform filter banks using the Hadamard-type tree structure, the required multiband responses appear 


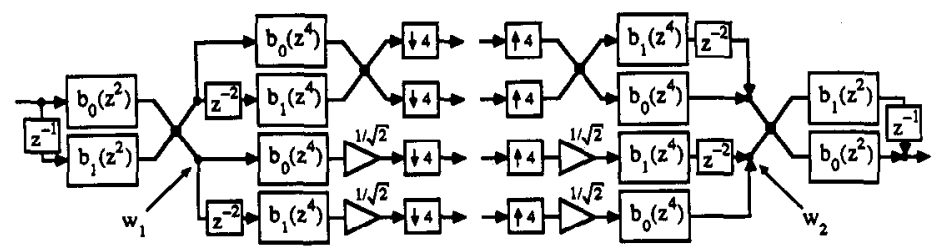

(a)

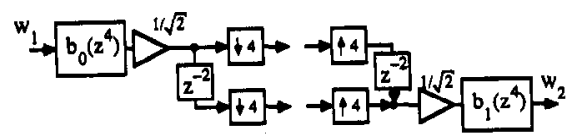

(b)

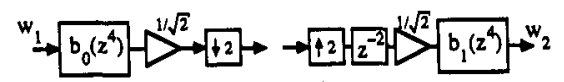

(c)

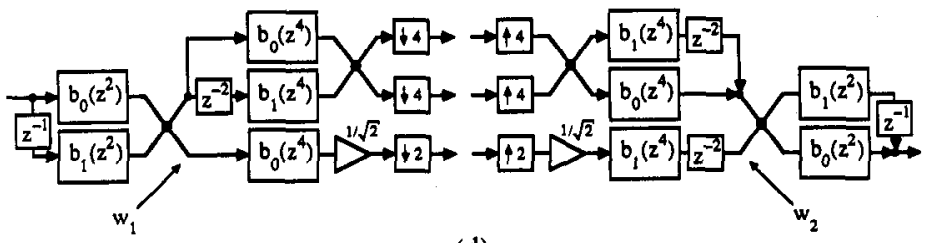

(d)

Fig. 19. Showing development of octave-band filter band.

naturally due to the periodicity of the amplitude responses of the decimated filter stages. Therefore, the filter design for these maximally decimated banks can be accomplished very simply by using, e.g., elliptic half-band low-pass/ high-pass transfer functions.

\section{APPENDIX \\ ProOF OF LEMMA 1}

From (33a), we need to show that if the $N \times N$ matrix $\boldsymbol{S} \triangleq \tilde{\boldsymbol{R}} \boldsymbol{Q R}$ is centro-skew-symmetric (i.e., skew-symmetric along the anti-diagonal), then

$$
\tilde{a}(z) \operatorname{Sa}(z)=0 \text {. }
$$

Let $s_{i, j}$ denote the $i, j$ th element of $S$. Centro-skew-symmetry then implies that $s_{i, j}=-s_{N-1-j, N-1-i}$. Thus by denoting

$$
a(z)=\left[\begin{array}{ll}
A_{0}(z) & A_{1}(z) \cdots A_{N-1}(z)
\end{array}\right]^{T}
$$

the scalar function of (A1) may be written as

$$
\begin{aligned}
& \tilde{\boldsymbol{a}}(z) \boldsymbol{S a}(z)=\sum_{i=0}^{N-1} \sum_{j=0}^{N-1-i} s_{i, j}\left[\tilde{A_{i}}(z) A_{j}(z)\right. \\
&\left.-\tilde{A}_{N-1-j}(z) A_{N-1-i}(z)\right] .
\end{aligned}
$$

We note the following observations:

(1) $N$ is a power of two, so let $N=2^{n}$.

(2) The all-pass function vector $a(z)$ derives from a binary tree structure (cf. Fig. 3), or equivalently from the Kronecker product formulation of (21) (repeated here for convenience):

$$
\boldsymbol{a}(z)=\left[\begin{array}{l}
a_{2 n-2}(z) \\
a_{2 n-1}(z)
\end{array}\right] \otimes\left[\begin{array}{l}
a_{2 n-4}(z) \\
a_{2 n-3}(z)
\end{array}\right] \otimes \cdots \otimes\left[\begin{array}{l}
a_{0}(z) \\
a_{1}(z)
\end{array}\right] .
$$

Thus each element $A_{i}(z)$ is the product of $n$ all-pass functions $a_{l}(z)$.

From observation (1) above, we may assign to each index $i$ an $n$-bit binary word. Note that the binary word corresponding to $N-1-i$ is obtained from that for $i$ by inverting each bit (i.e., using a ones' complement operation). Let us introduce the following notation:

$$
\begin{aligned}
{[i] } & =\text { binary word representation of } i \\
{[i] } & =\text { binary word representation of } N-1-i \\
& =\text { ones' complement of }[i]
\end{aligned}
$$

From observation (2), we identify a one-to-one correspondence between the bits in the index [i] of $A_{[i]}(z)$ and its all-pass factors $\left\{a_{l}(z)\right\}$ in (A2) and (A4). In particular, if the $l$ th bit of $[i]$ (counting from the right) is a zero, then $A_{[i]}(z)$ contains a factor $a_{2 l-2}(z)$. Similarly, if the $l$ th bit is a one, the corresponding factor is $a_{2 l-1}(z)$. We may then rewrite the factor in (A3) as

$$
\tilde{A}_{[i]}(z) A_{[j]}(z)-\tilde{A}_{[\bar{j}]}(z) A_{[\bar{i}]}(z)
$$

Now, any bits that $[i]$ and [ $j]$ have in common represent a cancellable factor. For example, if both [i] and [ $j]$ have the $l$ th bit as a one, then $\tilde{A}_{[i]}(z) A_{[j]}(z)$ has a factor of the form

$$
\tilde{a}_{2 l-1}(z) a_{2 l-1}(z)=1 .
$$

Similarly, $\tilde{A}_{[\bar{j} \mid}(z) A_{|i|}(z)$ has a factor $\tilde{a}_{2 l-2}(z) a_{2 l-2}(z)=1$. We thus observe: Any bits that $[i]$ does not have in common with $[j]$, it must have in common with $[\bar{j}]$, and likewise with $[j]$ and $[\bar{i}]$. After such, after removing factors of unity as in (A6) from both terms in (A5), we are left with an exact cancellation of remaining terms. Since (A3) is a weighted sum of terms as in (A5), then (A1) holds, which verifies the lemma. 


\section{REFERENCES}

[1] R. E. Crochiere and L. R. Rabiner, Multirate Digital Signal Processing. Englewood Cliffs, NJ: Prentice-Hall, 1983.

[2] A. Croisier, D. Estaban, and C. Galand, "Perfect channel splitting by use of interpolation/decimation/trec decomposition techniques," in Proc. 1976 Int. Conf. Inform. Sci. Syst., Patras, Greece, 1976.

[3] M. J. T. Smith and T. P. Barnwell, III, "Exact reconstruction techniques for tree-structured subband coders," IEEE Trans. Acoustics, Speech, Signal Processing, vol. ASSP-34, pp. 434-441, June 1986

[4] T. A. Ramstad and O. Foss, "Subband coder design using recursive quadrature mirror filters," in Signal Processing: Theories and Applications, M. Kunt and F. deCoulon (Eds.), Amsterdam, The Netherlands: North-Holland, 1980.

[5] P. C. Millar, "Recursive quadrature mirror filters-Criteria specification and design method," IEEE Trans. Acoustics, Speech, Signal Processing, vol. ASSP-33, pp. 413-420, Apr. 1985.

[6] P. L. Chu, "Quadrature mirror filter design for an arbitrary number of equal bandwidth channels," IEEE Trans. Acoustics, Speech, Signal Processing, vol. ASSP-33, pp. 203-218, Feb. 1985.

[7] A. Fettweis, J. A. Nossek, and K. Meerkötter, "Reconstruction of signals after filtering and sampling rate reduction," IEEE Trans. Acoustics, Speech, Signal Processing, vol. ASSP-33, pp. 893-902, Aug. 1985.

[8] K. Swaminathan and P. P. Vaidyanathan, "Theory and design of uniform DFT, parallel quadrature mirror filter banks," IEEE Trans. Circuits Syst., vol. CAS-33, pp. 1170-1191, Dec. 1986.

[9] P. P. Vaidyanathan and K. Swaminathan, "Uniform DSB QMF banks that are entirely free from aliasing," Tech., Rep., Calif. Inst. Tech., Pasadena, CA, May 1986.

[10] P. P. Vaidyanthan, "Theory and design of $M$-channel maximallydecimated quadrature mirror filters with arbitrary $M$, having the perfect reconstruction property," IEEE Trans. Acoustics, Speech, Signal Processing, vol. ASSP-35, pp. 476-492, Apr. 1987.

[11] M. Vetterli, "Filter banks allowing perfect reconstruction" Signal Processing, vol. 10, pp. 219-244, May 1986.

[12] M. J. T. Smith and T. P. Barnwell, III, "A unifying framework for analysis /synthesis systems based on maximally decimated filter banks," Proc. IEEE Int. Conf. Acoustics, Speech, and Signal Processing, pp. 521-524, Tampa, FL, Mar. 1985.

[13] H. Scheuermann and H. Göckler, "A comprehensive survey of digital transmultiplexing methods," Proc. IEEE, vol. 69, pp. 1419-1450, Nov. 1981.

[14] M. Bellanger and J. L. Daguet, "TDM-FDM transmultiplexer: Digital polyphase and FFT," IEEE Trans. Commun., vol. COM-22, pp. 1199-1204, Sept. 1974

[15] T. A. C. M. Claasen and W. F. G. Mecklenbrauker, "A generalized scheme for an all-digital time-division multiplex translator," IEEE Trans. Circuits Syst., vol. CAS-25, pp. 252-259, May 1978.

[16] Special Issue on TDM-FDM Conversion, IEEE Trans. Commun., vol. COM-26, May 1978.

[17] Special Issue on Transmultiplexers, IEEE Trans. Commun., vol. COM-30, July 1982.

[18] T. A. Ramstad, "Branch filtering using FIR and IIR complementary structures," in Digital Signal Processing, V. Cappellini and A. G. Constantinidis (Eds.), New York: Academic 1980.

[19] A. V. Oppenheim, Applications of Digital Signal Processing, Englewood Cliffs, NJ: Prentice-Hall, 1978, ch. 2.

[20] S. K. Mitra, Y. Neuvo, and P. P. Vaidyanathan, "Complementary IIR digital filter banks," in Proc. IEEE Int. Conf. Acoustics, Speech, and Signal Processing, Tampa, FL, pp. 529-532, 1985.

[21] A. Fettweis, H. Levin, and A. Sedlmeyer, "Wave digital lattice filters," Int. J. Circuit Theory Appl., vol. 2, pp. 203-211, 1974.

[22] L. Gazsi, "Explicit formulas for wave digital lattice filters," IEEE Trans. Circuits Syst., vol. CAS-32, pp. 68-88, Jan. 1985.

[23] R. Ansari and B. Liu, "A class of low-noise, computationally efficient recursive digital filters with applications to sampling rate alterations," IEEE Trans. Acoustics, Speech, Signal Processing, vol. ASSP-33, pp. 90-97, Feb. 1985.

[24] T. Saramäki, "On the design of digital filters as the sum of two all-pass filters," IEEE Trans. Circuits Syst, vol. CAS-32, pp. 1191-1193, Nov. 1985

[25] P. P. Vaidyanathan, S. K. Mitra, and Y. Neuvo, "A new approach to the realization of low-sensitivity IIR digital filters," IEEE Trans. Acoustics, Speech, Signal Processing, vol. ASSP-34, pp. 350-361, Apr. 1986

[26] A. H. Gray, Jr., and J. D. Markel, "Digital ladder and lattice filter synthesis," IEEE Trans. Audio Electroacoust., vol. AU-21, pp. 491-500, Dec. 1973

[27] S. K. Mitra and K. Hirano, "Digital all-pass networks," IEEE Trans. Circuits Syst., vol. CAS-21, pp. 688-700, Sept. 1974.

[28] P. P. Vaidyanathan, P. A. Regalia, and S. K. Mitra, "Design of doubly complementary IIR digital filters using a single complex allpass filter, with multirate applications," IEEE Trans. Circuits Syst., vol. CAS-34, pp. 378-389, Apr. 1987.
[29] F. A. Graybill, Matrices with Applications in Statistics, Belmont, CA: Wadsworth Intl. Group, 1983, ch. 8.

[30] N. Ahmed and K. Rao, Orthogonal Transforms for Digital Signal Processing. New York: Springer-Verlag, 1975.

[31] K. G. Beauchamp, Applications of Walsh and Related Functions. New York: Academic, 1984

[32] D. F. Elliot and K. R. Rao, Fast Transforms. New York: Academic, 1982

[33] B. J. Fino and V. R. Algazi, " $\Lambda$ unificd treatment of discrete fast unitary transforms," SIAM J. Comput., vol. 6, pp. 700-717, June 1971.

[34] K. R. Rao and N. Ahmed, “Complex BIFORE transform," Int. $J$. Syst. Sci., vol. 2, pp. 149-162, 1971

[35] N. Ahmed, K. R. Rao, and R. B. Schultz, "Fast complex BIFORE transform by matrix partitioning," IEEE Trans. Computers, pp. 707-710, June 1971.

[36] A. Cantoni and P. Butler, "Eigenvectors and eigenvalues of symmetric centrosymmetric matrices," Linear Algebra and its Applications, vol. 13, pp. 275-288, 1976.

[37] J. Makhoul, "On the eigenvalues of symmetric toeplitz matrices," IEEE Trans. Acoustics, Speech, Signal Processing, vol. ASSP-24, pp. 529-538, Dec. 1976.

[38] A. H. Gray, Jr., and J. D. Markel, "A computer program for designing digital elliptic filters," IEEE Trans. Acoustics, Speech, Signal Processing, vol. ASSP-24, pp. 529-538, Dec. 1976.

[39] S. K. Mitra, Y. Neuvo, and H. Roivainen, "Variable cutoff frequency digital IIR filters," in Proc. IASTED Int. Symp. Applied Signal Processing and Digital Filtering, Paris, France, June 1985.

[40] S. K. Mitra, A. J. Damonte, N. Fujii, and Y. Neuvo, "Tunable active crossover networks," J. Audio Eng. Soc., vol. 33, pp. 762-769, Oct. 1985.

[41] T. A. Ramstad, "Filter banks related to the cosine and Hadamard transforms," in Proc. Int. Conf. on Digital Signal Processing, pp. 292-296, Florence, Italy, Sept. 1981.

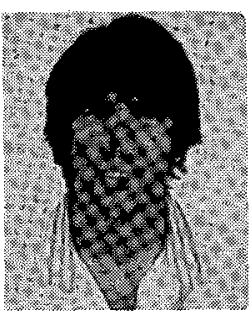

\section{承}

Phillip A. Regalia was born in Walnut Creek, $\mathrm{CA}$, in 1962 . He received the B. Sc. and M. Sc. degrees from the University of California at Santa Barbara in 1985 and 1987, respectively. At present he is a Ph.D. candidate at UCSB, where he works as a research assistant in the Signal Processing Laboratory.

His research interests include analog circuit theory and digital signal processing.

\section{承}

Sanjit K. Mitra (S'59-M'63-SM'69-F'74), for a photograph and biography please see page 886 of the August 1987 issue of this Transactions.

\section{W}

P. P. Vaidyanathan (S'80-M'83), for a photograph and biography please see page 23 of the January 1987 issue of this TRANSACTIONS.

\section{斗}

Markku K. Renfors (S'77-M'82), for a photograph and biography please see page 39 of the January 1987 issue of this TransaCtions.

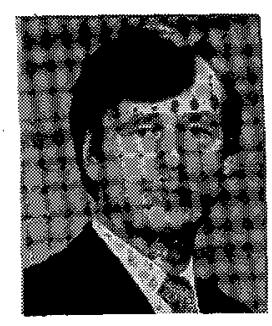

Yrjö Neuvo (S'70-M'74-SM'82) was born in Turku, Finland, on July 21, 1943. He received the Diploma Engineer and Licentiate of Technology degrees from the Helsinki University of Technology, Helsinki, Finland, in 1968 and 1971, respectively, and the Ph.D. degree in electrical engineering from Cornell University, Ithaca, NY, in 1974.

He held various research and teaching positions at the Helsinki University of Technology, the Academy of Finland, and Cornell University, from 1968 to 1976. Since 1976 he has been a Professor of Electrical Engineering at the Tampere University of Technology, Tampere, Finland. During the academic year 1981-1982 he was with the University of California, Santa Barbara, as a Visiting Professor. His main research interests are in the areas of digital filters and microcomputer systems.

Dr. Neuvo is a member of Phi Kappa Phi and the Finnish Academy of Technical Sciences. 